Technical Report

RAL-TR-2004-032

C C L R C

\title{
Numerical methods for large-scale nonlinear optimization
}

N I M Gould D Orban Ph L Toint

November 18, 2004 
(C) Council for the Central Laboratory of the Research Councils

Enquires about copyright, reproduction and requests for additional copies of this report should be addressed to:

Library and Information Services

CCLRC Rutherford Appleton Laboratory

Chilton Didcot

Oxfordshire OX11 0QX

UK

Tel: $+44(0) 1235445384$

Fax: $+44(0) 1235446403$

Email: library@rl.ac.uk

CCLRC reports are available online at:

http://www.clrc.ac.uk/Activity/ACTIVITY=Publications;SECTION=225;

ISSN 1358-6254

Neither the Council nor the Laboratory accept any responsibility for loss or damage arising from the use of information contained in any of their reports or in any communication about their tests or investigations. 


\title{
RAL-TR-2004-032
}

\section{Numerical methods for large-scale nonlinear optimization}

\author{
Nicholas I. M. Gould ${ }^{1,2,3}$, Dominique Orban ${ }^{4,5,6}$ and Philippe L. Toint ${ }^{7,8,9}$
}

\begin{abstract}
Recent developments in numerical methods for solving large differentiable nonlinear optimization problems are reviewed. State-of-the-art algorithms for solving unconstrained, bound-constrained, linearly-constrained and nonlinearly-constrained problems are discussed. As well as important conceptual advances and theoretical aspects, emphasis is also placed on more practical issues, such as software availability.
\end{abstract}

${ }^{1}$ Computational Science and Engineering Department, Rutherford Appleton Laboratory, Chilton, Oxfordshire, OX11 0QX, England, EU. Email: n.gould@rl.ac.uk

${ }^{2}$ Current reports available from "http://www.numerical.rl.ac.uk/reports/reports.html".

${ }^{3}$ This work was supported in part by the EPSRC grants GR/R46641 and GR/S42170.

${ }^{4}$ Department of Mathematics and Industrial Engineering, École Polytechnique, Montréal 2900, Bd E. Montpetit, H3T 1J4 Montréal, Canada.

Email: dominique.orban@polymtl.ca

${ }^{5}$ Current reports available from "http://www.ece.northwestern.edu/ orban/reports.html".

6 This work was supported in part by the NSERC grant RGPIN299010-04 and PIED grant 131FR88.

${ }^{7}$ Department of Mathematics, Facultés Universitaires ND de la Paix, 61, rue de Bruxelles, B-5000 Namur, Belgium, EU. Email : philippe.toint@fundp.ac.be

${ }^{8}$ Current reports available from "http://www.fundp.ac.be/ phtoint/pht/publications.html".

${ }^{9}$ This work was conducted in the framework of the Interuniversity Attraction Poles Programme of the Belgian Science Policy Agency.

Computational Science and Engineering Department

Atlas Centre

Rutherford Appleton Laboratory

Oxfordshire OX11 0QX

November 18, 2004. 


\section{Contents}

1 Introduction $\quad 4$

2 Large-scale unconstrained optimization 5

2.1 General problems . . . . . . . . . . . . . . . . . 5

2.1 .1 Computation of derivatives . . . . . . . . . . . 6

2.1.2 Computation of the step . . . . . . . . . . . . . 6

2.1.3 Practicalities ....................... 9

2.1.4 Software . . . . . . . . . . . . . . . . . 11

2.2 Least-squares problems . . . . . . . . . . . . . . . . . . . . . . . . . . . . . . . . . . . . . . . . . .

2.2 .1 Software . . . . . . . . . . . . . . . . . . . 12

2.3 Discretized problems . . . . . . . . . . . . . . . . . . . . 12

3 Large-scale bound-constrained optimization $\quad 13$

3.0.1 Active-set methods . . . . . . . . . . . . . . . . . . . . . 14

3.0.2 Gradient-projection methods . . . . . . . . . . . . . . 14

3.0 .3 Interior-point methods . . . . . . . . . . . . . . . . . 16

3.0.4 Practicalities . . . . . . . . . . . . . . . . . 18

3.0 .5 Software . . . . . . . . . . . . . . . . . . 18

4 Large-scale linearly-constrained optimization $\quad 19$

4.1 Equality-constrained quadratic programming . . . . . . . . . . . . . . . 19

4.2 General quadratic programming . . . . . . . . . . . . . . . . . 20

4.2.1 Active-set methods for general quadratic programming . . . . . . . 20

4.2.2 Interior-point methods for general quadratic programming . . . . . 22

4.2.3 Interior-point methods for convex quadratic programming . . . . . 24

4.2 .4 Practicalities . . . . . . . . . . . . . . . . 27

4.2 .5 Software . . . . . . . . . . . . . . . . . . 28

4.3 General linearly-constrained optimization . . . . . . . . . . . . . . 29

4.3 .1 Software . . . . . . . . . . . . . . . . . 30

5 Large-scale nonlinearly-constrained optimization $\quad 30$

5.1 Sequential linear and quadratic programming methods . . . . . . . . . . 30

5.2 SQP methods for equality-constrained problems . . . . . . . . . . . . . . . 30

5.3 SQP methods for the general problem . . . . . . . . . . . . . . 33

5.3.1 Sequential equality-constrained quadratic programming (SEQP) meth-

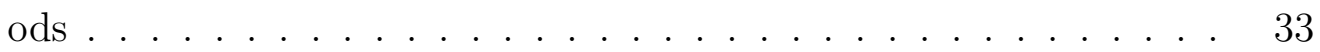

5.3.2 Sequential inequality-constrained quadratic programming (SIQP) methods .......................... 35

5.4 Interior-point methods for nonlinear programs . . . . . . . . . . . . 37

5.4.1 Practicalities . . . . . . . . . . . . . . . . . . 42

5.4.2 Problems with equilibrium constraints . . . . . . . . . . . 43 
5.4.3 General convex programs . . . . . . . . . . . . . . . . . . 44

5.4 .4 Software . . . . . . . . . . . . . . . . . 45

6 Conclusion $\quad 46$ 


\section{Introduction}

Large-scale nonlinear optimization is concerned with the numerical solution of continuous problems expressed in the form

$$
\underset{x \in \mathbb{R}^{n}}{\operatorname{minimize}} f(x) \text { subject to } c_{\mathcal{E}}(x)=0 \text { and } c_{\mathcal{I}}(x) \geq 0,
$$

where $f: \mathbb{R}^{n} \rightarrow \mathbb{R}, c_{\mathcal{E}}: \mathbb{R}^{n} \rightarrow \mathbb{R}^{n_{\mathcal{E}}}$ and $c_{\mathcal{I}}: \mathbb{R}^{n} \rightarrow \mathbb{R}^{n_{\mathcal{I}}}$ are smooth and $n$, and possibly $n_{\mathcal{E}}$ and/or $n_{\mathcal{I}}$, are large. Here, the components of the vector $x$ are the variables, $f(x)$ is the

objective function and the components of the vectors $c_{\mathcal{E}}(x)$ and $c_{\mathcal{I}}(x)$ are the constraint functions. Such problems arise throughout science, engineering, planning and economics. Fortunately algorithmic development and theoretical understanding generally continue to keep apace with the needs of such applications.

Our purpose in this paper is to review recent developments, with an emphasis on discussing state-of-the-art methods for various problem types fitting within the broad definition (1). As the title indicates, we will focus on nonlinear problems, that is on problems for which at least one of the functions involved is nonlinear - although many of the methods for linear programming are variants of those in the nonlinear case, extra efficiencies are generally possible in this first case, and the general state-of-the-art is to be able to solve linear problems perhaps ten times larger than nonlinear ones (Bixby, Fenlon, $\mathrm{Gu}$, Rothberg and Wunderling, 2000). We shall also mostly be concerned with large problems, that is, at the time of writing, those involving of the order of 100,000 variables and perhaps a similar number of constraints. However, we accept that this estimate may be too conservative for some problem classes - for instance larger quadratic programs can certainly be solved today. Moreover, structure plays an important role in the size of problems that can be tackled: large sparse or partially separable cases are easier to handle than dense ones. Finally, the definition of a large problem may also depend on the hardware used, although this effect is less visible than in the past because of the remarkable evolution of personal computers in terms of memory processing power.

We will not review the history of the field here, but refer the interested reader to Gould and Toint (2004b) for a brief perspective and a discussion of the reasons why this mature research domain remains so active and why this will likely continue for some time. The field has acquired a vast literature, and there have been numerous attempts to synthesize various aspects of it in books, such as those by Bertsekas (1995), Bonnans, Gilbert, Lemaréchal and Sagastizábal (1997), Dennis and Schnabel (1983), Fletcher (1981), Gill, Murray and Wright (1981), Moré and Wright (1993), Nash and Sofer (1990), Nocedal and Wright (1999), Conn, Gould and Toint (2000a), in volumes of conference proceedings, such as those edited by Coleman and Li (1990), Leone, Murli, Pardalos and Toraldo (1998), Di Pillo and Gianessi (1996, 1999), Di Pillo and Murli (2003), Hager, Hearn and Pardalos (1994), Spedicato (1994), Yuan (1998), in survey articles, like those given by Conn, Gould and Toint (1994, 1996), Fletcher (1987b), Forsgren, Gill and Wright (2002), Gould (2003), Marazzi and Nocedal (2001), Nash (2000b) and, in this series, by Boggs and Tolle (1995), Lewis and Overton (1996), Nocedal (1992), Powell (1998), Todd (2001), and Wright (1992). 
The paper is structured as follows. Sections of the paper deal with problem classes: $\S 2$ covers unconstrained problems, while bound- and linearly-constrained problems are reviewed in $\S 3$ and $\S 4$, respectively, and $\S 5$ considers general nonlinearly-constrained cases. In each of these sections, subsections refer to method classes, allowing the interested reader to focus on these across different problem types. In particular, we discuss linesearch and trust-region methods successively. We conclude most sections with a paragraph on practicalities and a paragraph on software. Final comments are made in $\S 6$.

\section{Large-scale unconstrained optimization}

\subsection{General problems}

Although general unconstrained optimization problems (i.e., problems where $\mathcal{E}$ and $\mathcal{I}$ are empty in (1)) arise relatively infrequently in practice - non-linear least-squares problems (see §2.2) being a notable exception - a brief discussion of methods for unconstrained optimization is useful if only for understanding those for problems involving constraints. For a fuller discussion see Nocedal $(1992,1997)$. While hybrids are possible, the essential distinction over the past 35 years has been between the linesearch and trust-region approaches.

Given an estimate $x_{k}$ of an unconstrained minimizer of $f(x)$, both paradigms rely on simple (differentiable) models $m_{k}(d)$ of the objective function $f\left(x_{k}+d\right)$. For linesearch methods $m_{k}$ will normally be convex while this is not required in the trust-region case; for both it is usually important that $m_{k}(0)=f\left(x_{k}\right)$ and $\nabla_{x} m_{k}(0)=\nabla_{x} f\left(x_{k}\right)$. Given a suitable model, a model-improving approximate minimizer $d_{k}$ is computed. In the trust-region case, possible unboundedness of the model is naturally handled by the trust-region constraint $\|d\| \leq \Delta_{k}$ for some $\Delta_{k}>0$. Since the model is only a local representation of the objective function, it is possible that predicted improvements in $f$ may not actually be realized. Linesearch methods account for this by retracting the step along $d_{k}$ so that $x_{k}+\alpha_{k} d_{k}$ gives an improvement in $f$. By contrast, trust-region methods reject steps for which there is poor agreement between the decrease in $m_{k}$ and $f$, and rely on a reduction of the radius $\Delta_{k+1}$, and thus a re-computation of $d_{k+1}$, to ensure improvement. The mechanics of finding the step-size $\alpha_{k}$ for linesearch methods (Hager and Zhang, 2003, Moré and Thuente, 1994) and adjusting the radius $\Delta_{k}$ in trust-region methods (Conn et al., 2000a, §17.1) has been much studied, and can have a significant effect on the performance of an algorithm. But overall the dominant computational cost of both classes of algorithms is in evaluating the values and required derivatives of $f$ and in computing the step $d_{k}$; the cost of evaluating $f$ often dominates in simulation-based applications or industry problems, but quite rarely in problems defined in commonly-occurring modeling languages such as AMPL (Fourer, Gay and Kernighan, 2003) or GAMS (Brooke, Kendrick and Meeraus, 1988). 


\subsubsection{Computation of derivatives}

In the early days, researchers invested much effort in finding methods with modest derivative requirements. Typically function values and, sometimes, gradients were available, but second derivatives frowned upon. The advent of automatic differentiation (Griewank, 2000) and (group) partial separability (Griewank and Toint, 1982b, Conn, Gould and Toint, 1990) has somewhat altered this position at least amongst researchers, and now methods that are designed to exploit second derivatives (or good approximations thereof) are commonplace. But it is arguable that such new methods have not been as widely used by practitioners as might have been hoped, often because application codes capable of computing function values are unnameable to automatic differentiation for a variety of reasons, size and unavailability of the source-code being two common complaints. Indeed, there are still many practitioners who prefer methods that avoid derivatives at all (Powell, 1998), although

such methods are usually only appropriate for small-scale problems (but see Colson and Toint, 2003, or Price and Toint, 2004, for recent attempts to extend these techniques to large-scale cases).

Automatic differentiation offers the possibility of computing gradients and Hessianvector products at a few times the cost of a function value (Griewank, 2000). Tools for automatic differentiation are available both as stand-alone software or as part of modeling languages (AMPL and GAMS being good examples). Partial separability allows the computation of finite-difference gradients at a similar cost if only function values are available, and the same for Hessians if (structured) gradients can be found (Conn et al., 1990). Moreover accurate structured secant approximations to second derivatives can be computed (Griewank and Toint, 1982b), and this allows one to approximate gradients (by finite-differences) and Hessians (by secant formulae) just given function values if the problem functions are partially separable and the structure specified (Conn, Gould and Toint, 1996).

Note that these comments on evaluating derivatives are of interest not only for unconstrained problems, but also for most of the other problems that are discussed in this paper. In the constrained case, the derivative of the constraint and Lagrangian functions will also be concerned, and the techniques to compute them are similar to what we have just mentioned.

\subsubsection{Computation of the step}

Even if function and derivative values are available, in general the cost of computing the step $d_{k}$ may be significant if the problem involves a large number of variables. This computation often follows the following line: if $H_{k}$ is a symmetric positive-definite approximation to $\nabla_{x x} f\left(x_{k}\right)$, if the quasi-Newton (QN) model

$$
m_{k}(d)=f\left(x_{k}\right)+d^{T} \nabla_{x} f\left(x_{k}\right)+\frac{1}{2} d^{T} H_{k} d
$$


is used, and if the minimizer of this model is sought, the resulting step $d_{k}$ satisfies the QN equations

$$
H_{k} d_{k}=-\nabla_{x} f\left(x_{k}\right) .
$$

Since $H_{k}$ is positive definite, realistic solution options include a (sparse) Cholesky factorization of $H_{k}$ or application of the (preconditioned) conjugate-gradient (CG) method (Hestenes and Stiefel, 1952). The former may be unviable if the factors fill-in significantly, but is capable of giving a numerical solution with small relative error. The latter is more flexible - rather than needing $H_{k}$, it merely requires a series of products $H_{k} p$ for given vectors $p$ (and possibly preconditioned residuals $r=P_{k}^{-1} g$ for some suitable symmetric preconditioner $P_{k}$ ), and thus is better equipped for automatic differentiation or finitedifference gradient approximations $\left(\nabla_{x} f\left(x_{k}+\epsilon p\right)-\nabla_{x} f\left(x_{k}\right)\right) / \epsilon$ for small $\epsilon$ - but less likely to be able to compute highly accurate numerical solutions of (3). When the approximation $H_{k}$ is indefinite, it may be modified during factorization (Schlick, 1993) or as the CG process proceeds (Nash, 1984) to restore definiteness. Alternatively, the CG method may be terminated appropriately as soon as one of the products $H_{k} p$ in the $\mathrm{CG}$ method reveals negative curvature (Dembo and Steihaug, 1983) or even continued in the subspace of positive curvature whilst gathering negative curvature information (Gould, Lucidi, Roma and Toint, 2000b).

A significant breakthrough for large-scale unconstrained optimization occurred in the early 1980s with the advent of truncated-QN methods (Dembo, Eisenstat and Steihaug, 1982). Here, rather than requiring that $d_{k}$ satisfies (3), instead $d_{k}$ is asked to satisfy

$$
\left\|H_{k} d_{k}+\nabla_{x} f\left(x_{k}\right)\right\| \leq \eta_{k}\left\|\nabla_{x} f\left(x_{k}\right)\right\|
$$

where $0<\eta_{k}<1$ and $\eta_{k} \rightarrow 0$ if $\nabla_{x} f\left(x_{k}\right) \rightarrow 0$. This is helpful for use in conjunction with CG methods, since one could anticipate being able to satisfy (4) after few CG iterations for modest values of $\eta_{k}$. But more significantly - and perhaps overlooked by those who view CG as simply a method for solving linear systems - the iterates $\left\{d_{k, j}\right\}_{j \geq 0}$ generated by the CG method from $x_{k}$ have two further fundamental properties. Firstly, by construction each successive CG step further reduces the model, that is $m_{k}\left(d_{k, j+1}\right)<m_{k}\left(d_{k, j}\right)$ for $j \geq 0$. Secondly an appropriate norm of the CG iterates increases at each step, that is $\left\|d_{k, j+1}\right\|>$ $\left\|d_{k, j}\right\|$ for $j \geq 0$ (Steihaug, 1983). This enables one to construct globally convergent linesearch (Dembo and Steihaug, 1983) and trust-region (Steihaug, 1983, Toint, 1981) truncated Newton methods, i.e. methods that converge to local solutions from arbitrary starting points. In the linesearch case, $d_{k}$ is chosen as the first $d_{k, j}$ for which (4) is satisfied, unless negative curvature is discovered when computing the required product $H_{k} p$ at $\mathrm{CG}$ iteration $j$, in which case either the steepest descent direction $-\nabla_{x} f\left(x_{k}\right)$ (when $j=0$ ) or the current CG approximation $d_{k, j-1}$ (when $j>0$ ) may be used instead (Dembo and Steihaug, 1983). For the trust-region case, such methods should be stopped on the trustregion boundary if $\left\|d_{k, j}\right\|>\Delta_{k}$ or negative-curvature is discovered, since once the CG iterates leave the trust-region they will not return (Steihaug, 1983). By judicious control of $\eta_{k}$ in (4), such methods may also be shown to be superlinearly convergent under reasonable conditions on the approximation $H_{k}$ to $\nabla_{x x} f\left(x_{k}\right)$. 
In the trust-region case, an accurate solution of the model problem needs to account for the trust region constraint $\|d\| \leq \Delta_{k}$. When $H_{k}$ is positive semi-definite, the strategy of truncating the CG iteration on the trust-region boundary (Steihaug, 1983, Toint, 1981) ensures a model decrease which is at least half as good as the optimal decrease (Yuan, 2000). For indefinite $H_{k}$ this is not so. Although there are excellent methods for solving the problem in the small-scale case (Moré and Sorensen, 1983), these rely on being able to solve a (small) sequence of linear systems with coefficient matrices $H_{k}+\sigma_{k, l} I$ for given $\sigma_{k, l} \geq 0$, and thus implicitly on being able to factorize each coefficient matrix. Since this may be expensive or even impossible in the large-scale case, an alternative is to note that the CG and Lanczos methods compute different bases for the same Krylov space, and that after $j$ steps of the Lanczos method, $Q_{k, j}^{T} H_{k} Q_{k, j}=T_{k, j}$ where the columns of the $n$ by $j$ matrix $Q_{k, j}$ are orthonormal and $T_{k, j}$ is tridiagonal. Thus if we seek an approximation to the solution of the trust-region problem in the range of the expanding matrix $Q_{k, j}$, we may compute

$$
d_{k, j}=Q_{k, j} h_{k, j}, \quad \text { where } h_{k, j}=\underset{\|h\| \leq \Delta_{k}}{\arg \min } e_{1}^{T} Q_{k, j}^{T} \nabla_{x} f\left(x_{k}\right) e_{1}^{T} h+\frac{1}{2} h^{T} T_{k, j} h,
$$

where $e_{1}=[1,0,0, \ldots, 0]^{T}$. Since $T_{k, j}$ is tridiagonal, we may reasonably factorize $T_{k, j}+$ $\sigma_{k, j, l} I$, and thus the earlier Moré-Sorensen method is now applicable (Gould, Lucidi, Roma and Toint, 1999). The Lanczos iteration may be truncated in a similar way to (4), preconditioning may be readily incorporated, and the resulting so-called GLTR method has been used as a subproblem solver in a number of large-scale optimization packages (Byrd, Gould, Nocedal and Waltz, 2004a, Gould, Orban and Toint, 2003a). Other iterative methods for the exact minimization of (2) within the trust-region have been proposed (Hager, 2001, Rendl and Wolkowicz, 1997, Sorensen, 1997), but as far as we are aware they have not been used in truncated form.

Another popular and effective method is the limited-memory secant approach (Gilbert and Lemaréchal, 1989, Liu and Nocedal, 1989, Nocedal, 1980). Secant methods maintain Hessian approximations by sequences of low-rank updates, each using a pair of vectors $\left(d_{k}, y_{k}\right)$, where $y_{k}=\nabla_{x} f\left(x_{k+1}\right)-\nabla_{x} f\left(x_{k}\right)$, to satisfy the secant condition $H_{k} d_{k}=y_{k}$ (Nocedal and Wright, 1999, §2.2). Noting the success of (particularly) the BFGS secant method for small-scale computation, and recognising that such methods are generally inappropriate for large problems because the generated matrices are almost invariably dense, the idea of limited memory methods is simply to use no more than $m$ pairs $\left\{\left(d_{j}, y_{j}\right)\right\}_{j=k-m+1}^{k}$ to generate a secant approximation from a given, easily invertible initial matrix. If $m$ is small, application of the resulting limited-memory approximation $H_{k}$ or its inverse to a given vector may be performed extremely efficiently (Byrd, Nocedal and Schnabel, 1994). Although this approach is perhaps most natural in a linesearch framework-because the QN direction $-H_{k}^{-1} \nabla_{x} f\left(x_{k}\right)$ is easy to obtain - it may also be used in a trust-region one (Burke and Weigmann, 1997, Kaufman, 1999).

Since estimating $H_{k}$ directly by secant methods is likely out of the question for large problems, an alternative we have already briefly mentioned is to exploit problem struc- 
ture, and most especially partial separability, to obtain good Hessian approximations. By definition, a partially separable function has the form $f(x)=\sum_{i} f^{(i)}(x)$, where each element $f^{(i)}$ has a large invariant subspace. Thus it is reasonable to approximate $\nabla_{x x} f(x)$ by $\sum_{i} H^{(i)}$, where each $H^{(i)}$ approximates the low-rank element Hessian $\nabla_{x x} f^{(i)}(x)$. So-called partitioned QN methods (Griewank and Toint, 1982c) use suitable secant formulae to build (often highly accurate) approximations $H^{(i)}$. Although the resulting $H_{k}=\sum_{i} H_{k}^{(i)}$ may not be as easily inverted as, say, that from a limited-memory method, it often gives more accurate approximations, and has been used with great success within a truncated CG framework (Conn et al., 1990).

The final major class of methods are nonlinear variants of the CG method. Briefly, these methods aim to mimic the linear CG approach, and the step $d_{k}$ is updated every iteration so that

$$
d_{k+1}=-\nabla_{x} f\left(x_{k}\right)+\beta_{k} d_{k}
$$

for some appropriate scalar $\beta_{k}$. Such methods have a long pedigree (Fletcher and Reeves, 1964, Gilbert and Nocedal, 1992, Polak and Ribiere, 1969, Powell, 1977). Early methods chose $\beta_{k}$ using formulae derived from the linear CG method, but sometimes subsequent steps tended to be closely dependent. A number of modifications have been proposed to avoid this defect, many of them resorting to steps in, or close to, the steepest-descent direction. The most successful recent methods (Dai and Yuan, 2000, Hager and Zhang, 2003) achieve this seamlessly, and additionally use linesearches with weak step-size acceptance criteria.

\subsubsection{Practicalities}

Despite the large number of papers devoted to large-scale unconstrained optimization, it is quite difficult to find comparisons between the various approaches proposed. A 1991 survey by Nash and Nocedal (1991) compares the limited-memory L-BFGS method (Liu and Nocedal, 1989) with both the (early) Polak-Ribière nonlinear CG method (Polak and Ribiere, 1969) and a truncated-Newton method in which Hessian-vector products are obtained by differences. Although the results are mixed, the truncated-Newton approach seems preferable for problems well-approximated by a quadratic while L-BFGS appears best for more nonlinear problems. The nonlinear CG method is often best in terms of time, but requires more function evaluations. A contemporary survey by Gilbert and Nocedal (1992) which compares various nonlinear CG methods indicates there is little to choose between various variants on the Polak-Ribière theme. However, while the test problems might have been large by 1990 standards, they are certainly not by today's. The only recent comparison we are aware of is that by Hager and Zhang (2003), in which their modern nonlinear CG method is compared with L-BFGS and Gilbert and Nocedal's (1992) improvement to Polak-Ribière. At least on the basis of these tests, modern nonlinear CG appears to be the method of choice if second derivatives are unavailable. However, we should exercise some caution as again the problems were not really large by today's standard, nor do we know how second-derivative-based truncated-Newton fit into the picture. 
Two other issues are vital for good performance of many of the methods we have discussed. The first is preconditioning, where beyond very simple ideas such as diagonal or band scaling using Hessian terms (Conn et al., 1990), little has been done excepting to use standard incomplete factorization ideas from numerical linear algebra - Lin and Moré's (1999a) memory-conserving incomplete factorization is widely used in optimization circles. One interesting idea is to use a limited-memory approximation to $H_{k}$ to precondition the next subproblem $H_{k+1}$ (Morales and Nocedal, 2000), although more experience is needed to see if this is generally applicable.

The second important advance is based on the observation that while there should be some overall monotonically reducing trend of function values in algorithms for minimization, this is not necessary for every iteration (Grippo, Lampariello and Lucidi, 1986). Non-monotonic methods for unconstrained problems were first proposed in a linesearch framework (Grippo, Lampariello and Lucidi, 1989), and have been observed to offer significant gains when compared with their monotone counterparts (Toint, 1996). The same is true in a trust-region context (Deng, Xiao and Zhou, 1993, Toint, 1997), and many algorithms now offer non-monotonic variants (Gould et al., 2003a).

Another technique that exploits the potential benefits of non-monotonicity uses the idea of filters. Inspired from multi-objective optimization and originally intended by Fletcher and Leyffer (2002) for constrained problems (see $\S 5.1$ below), the aim of a filter is to allow conflicting abstract objectives within the design of numerical algorithms. To understand the idea, consider an abstract situation where one attempts to simultaneously reduce two potentially conflicting objectives $\theta_{1}(x)$ and $\theta_{2}(x)$. A point $x$ is then said to dominate another point $y$ if and only if $\theta_{i}(x)<\theta_{i}(y)$ for $i=1$ and 2 (this definition can obviously be generalized to more than two conflicting objectives). Remembering a dominated $y$ is of little interest when aiming to reduce both $\theta_{1}$ and $\theta_{2}$ since $x$ is at least as good as $y$ for each objective. Obviously, an algorithm using this selection criterion should therefore store some or all pairs $\left(\theta_{1}, \theta_{2}\right)$ corresponding to successful previous iterates.

It turns out that this concept allows the design of new non-monotonic techniques for unconstrained minimization. For convex problems, we know that finding the (unique) minimizer is equivalent to finding a zero of the gradient. This in turn may be viewed as the (potentially conflicting) objective of zeroing each of the $n$ gradient components $\left[\nabla_{x} f(x)\right]_{i}(i=1, \ldots, n)$. One may therefore decide that a new trial point $x_{k}+d_{k}$ is not acceptable as a new iterate only if it is dominated by $x_{p}$, one of (a subset of) the previous iterates, in the sense that

$$
\left|\left[\nabla_{x} f\left(x_{p}\right)\right]_{i}\right|<\left|\left[\nabla_{x} f\left(x_{k}+d_{k}\right)\right]_{i}\right|
$$

for all $i=1, \ldots, n$, which corresponds to the choice $\theta_{i}(x)=\left|\left[\nabla_{x} f\left(x_{k}\right)\right]_{i}\right|(i=1, \ldots, n)$. The subset of previous iterates $x_{p}$ for which the values of the gradient components are remembered and this comparison conducted is called the "filter" and is maintained dynamically. If $x_{k}+d_{k}$ is not acceptable according to (5), it can still be evaluated using the more usual trust-region technique, which then guarantees that a step is eventually acceptable and that a new iterate can be found. Unfortunately, this technique might prevent 
progress away from a saddle point for nonconvex problems, in which case an increase in the gradient components is warranted. The filter mechanism is thus modified to dynamically disregard the filter in these cases. The details of the resulting algorithm are described by Gould, Sainvitu and Toint (2004b), where encouraging numerical results are also reported on both small- and large-scale problems.

\subsubsection{Software}

There is a lot of easily-available software for unconstrained minimization. Here, and later, we refer the reader to the on-line software guides

http://www-fp.mcs.anl.gov/otc/Guide/SoftwareGuide/ and http://plato.asu.edu/guide.html,

by Moré and Wright, and Mittelmann and Spellucci, respectively. Of the methods discussed in this section, TN/TNBC (Nash, 1984) is a truncated CG method, LBFGS (Liu and Nocedal, 1989) is a limited-memory QN method, VE08 (Griewank and Toint, 1982c) is a partitioned QN method, and CG+ (Gilbert and Nocedal, 1992) and CG_DESCENT (Hager and Zhang, 2003) are nonlinear CG methods. In addition, software designed for more general problems - for example IPOPT, KNITRO, LANCELOT, LOQO and TRON - is often more than capable when applied in the unconstrained case.

\subsection{Least-squares problems}

Nonlinear least-squares problems, for which

$$
f(x)=\sum_{i=1}^{m} f_{i}^{2}(x)
$$

are perhaps the major source of really unconstrained problems. In particular, large sets of nonlinear equations, parameter estimation in large dynamical systems and free surface optimization often result in sizeable and difficult instances (see Gould and Toint, 2004b, for examples). Methods for solving problems of this type follow the general trends of $\S 2.1$, but specifically exploit the special form of the objective function to select-sometimes adaptively (Dennis, Gay and Welsh, 1981) - between the "full QN" model, where the matrix $H_{k}$ in (2) is chosen to approximate the Hessian

$$
\nabla_{x x} f(x)=J(x)^{T} J(x)+\sum_{i=1}^{m} f_{i}(x) \nabla_{x x} f_{i}(x)
$$

(where $J(x)$ is the $m \times n$ matrix whose rows are the gradients $\nabla_{x} f_{i}(x)$ ), and the cheaper "Gauss-Newton" model for which $H_{k}=J(x)^{T} J(x)$. Furthermore, algorithmic stopping criteria can be adapted to exploit the special structure of $\nabla_{x} f(x)$ and the fact that zero provides an obvious lower bound on the value of the objective function. 
Apart from the contributions of Al-Baali (2003) on dedicated QN updates, the work of Lukšan $(1993,1994,1996)$ on incorporating iterative linear algebra techniques in trustregion algorithms for nonlinear least-squares and that of Gulliksson, Söderkvist and Wedin (1997) on handling weights (and constraints), there has been little recent research in this area. Of course, most new ideas applicable to general unconstrained optimization may also be applied in the nonlinear least-squares case.

This is in particular the case for filter methods. In this context, the idea is to associate one filter objective $\theta_{i}(x)$ with each residual, i.e., $\theta_{i}(x)=f_{i}(x)(i=1, \ldots, m)$, or perhaps with the norm of a block of residuals, i.e., $\theta_{i}(x)=\left(\sum_{j \in J_{i}} f_{j}^{2}(x)\right)^{\frac{1}{2}}$ for some $J_{i} \subset\{1, \ldots, m\}$. Details along with proofs of convergence are given by Gould, Leyffer and Toint (2004a). Such ideas may be trivially extended to incorporate inequality constraints, which then provides practical means for solving the nonlinear feasibility problems (that is, to find a solution to a set of nonlinear equality and inequality constraints in the least-squares sense). Numerical efficiency and reliability is considered by Gould and Toint (2003a).

\subsubsection{Software}

The only dedicated large-scale nonlinear least-squares packages we are aware of are the sparsity-exploiting SPRNLP (Betts and Frank, 1994), VE10 (Toint, 1987) which uses the obvious partially-separable structure of such problems, and the filter-based code FILTRANE from the GALAHAD library (Gould et al., 2003a). Of course much general-purpose software is applicable to nonlinear least-squares problems.

\subsection{Discretized problems}

In practice, many large-scale finite-dimensional unconstrained optimization problems arise from the discretization of those in infinite-dimensions, a primary example being leastsquares parameter identification in systems defined in terms of either ordinary or partial differential equations. The direct solution of such problems for a given discretization yielding the desired accuracy is often possible using general packages for large-scale numerical optimization (see §2.1). However, such techniques rarely make use of the underlying infinite-dimensional nature of the problem, for which several levels of discretization are possible, and thus such approach rapidly becomes cumbersome. Multi-scale (sometimes known as multi-level) optimization aims at making explicit use of the problem structure in the hope of improving efficiency and, possibly, enhancing reliability.

Using differing scales of discretization for an infinite-dimensional problem is not a new idea. An obvious simple "mesh refinement" approach is to use coarser grids in order to compute approximate solutions which can then be used as starting points for the optimization problem on a finer grid (Griewank and Toint, 1982a, Bank, Gill and Marcia, 2003, Betts and Erb, 2003, Benson, McInnes, Moré and Sarich, 2004). However, potentially more efficient techniques are inspired from the multigrid paradigm in the solution of partial differential equations and associated systems of linear algebraic equations (Brandt, 1977, Bram- 
ble, 1993, Hackbusch, 1995, Briggs, Henson and McCormick, 2000), and have only been discussed relatively recently in the optimization community. Contributions along this direction include the "generalized truncated Newton algorithm" presented in Fisher (1998), and those by Moré (2003), Nash (2000a) and Lewis and Nash (2002, 2004). The latter three papers present the description of MG/OPT, a linesearch-based recursive algorithm, an outline of its convergence properties and impressive numerical results. The generalized truncated Newton algorithm and MG/OPT are very similar and, like many linesearch methods, naturally suited to convex problems, but their extension to nonconvex cases is also possible. Very recently, Gratton, Sartenaer and Toint (2004) have proposed a recursive multi-scale trust-region algorithm (RMTR) which fits nonconvex problems more naturally and is backed by a strong convergence theory. The main idea of all the methods mentioned here is to (recursively) exploit the cheaper optimization on a coarse mesh to produce steps that significantly decrease the objective function on a finer mesh, while of course continuing to benefit from mesh refinement for obtaining good starting points. In principle, low frequency components of the problem solution (suitably prolongated in the original infinite-dimensional space of interest) are determined by the coarse mesh calculations, and optimizing on the fine mesh then only fixes high frequency components.

While the idea appears to be very powerful and potentially leads to the solution of very large-scale problems, the practical algorithms that implements them are still mostly experimental. Preliminary numerical results are encouraging, but the true potential of these methods will only be confirmed by continued success in the coming years.

A second interesting approach to very large problems arising from continuous applications it to look at other ways to simplify them and make them more amenable to classical optimization techniques. For instance, Arian, Fahl and Sachs (2000) and Fahl and Sachs (2003) investigate the use of reduced-order models (using proper orthogonal decomposition techniques) in the framework of trust-region algorithms, and apply this technique to fluid-mechanics problems. Note that model simplification of that type can also be thought of as a recursive process, although not immediately based on discretization. The idea is thus close in spirit to the proposals described above. Again, the practical power of this approach, although promising at this stage, is still the object of ongoing evaluation.

\section{Large-scale bound-constrained optimization}

In the simplest of constrained optimization problems, we seek the minimizer of $f(x)$ within a feasible box, $\Omega=\{x \mid l \leq x \leq u\}$ for given (possibly infinite) lower and upper bounds $l$ and $u$. Without loss of generality, we assume that $l_{i}<u_{i}$ for all $i=1, \ldots, n$. It has been argued that all unconstrained problems should actually include simple bounds to prevent bad effects of computer arithmetic such as overflows, and certainly many real problems have simple bounds to prevent unreasonable or physically-impossible values. 


\subsubsection{Active-set methods}

Early methods for this problem tended to be of the active-set variety. The active set at $x$ is $\mathcal{A}(x)=\mathcal{L}(x) \cup \mathcal{U}(x)$, where $\mathcal{L}(x)=\left\{i \mid x_{i}=l_{i}\right\}$ and $\mathcal{U}(x)=\left\{i \mid x_{i}=u_{i}\right\}$. Trivially, if $x_{*}$ is a (local) minimizer of $f$ within $\Omega, x_{*}$ is a (local) minimizer of $f(x)$ subject to $x_{i}=l_{i}, i \in \mathcal{L}\left(x_{*}\right)$ and $x_{i}=u_{i}, i \in \mathcal{U}\left(x_{*}\right)$. Active set methods aim to predict $\mathcal{L}\left(x_{*}\right)$ and $\mathcal{U}\left(x_{*}\right)$ using suitably chosen disjoint sets $\mathcal{L}, \mathcal{U} \subseteq\{1, \ldots, n\}$. Given $\mathcal{L}$ and $\mathcal{U}$, a typical method will aim to (approximately)

$$
\begin{aligned}
& \operatorname{minimize} f(x) \\
& \text { subject to } x_{i}=l_{i}, i \in \mathcal{L} \text { and } x_{i}=u_{i}, i \in \mathcal{U}
\end{aligned}
$$

such a calculation is effectively an unconstrained minimization over the variables $\left(x_{i}\right), i \notin$ $\mathcal{A}=\mathcal{L} \cup \mathcal{U}$, and thus any of the methods mentioned in $\S 2$ is appropriate. Of course the predictions $\mathcal{L}$ and $\mathcal{U}$ may be incorrect, and the "art" of active-set methods is to adjust the sets as the iteration proceeds either by adding variables which violate one of their bounds or by removing those for which further progress is predicted - the same idea is possible (and indeed used) to deal with more general inequality constraints. See Gill et al. $(1981, \S 5.5)$ or Fletcher $(1987 a, \S 10.3)$ for more details. Especially effective methods for the quadratic programming case, for which $f$ is quadratic, have been developed (Coleman and Hulbert, 1989).

The main disadvantage of (naive) active-set methods for large-scale problems is the potential worst-case complexity in which each of the possible $3^{n}$ active sets is visited before discovering the optimal one. Although it is possible to design active-set methods for the simple-bound case that are capable of making rapid changes to incorrect predictions (Facchinei, Judice and Soares, 1998), it is now more common to use gradient-projection methods.

\subsubsection{Gradient-projection methods}

The simplest gradient-projection algorithm (Bertsekas, 1976, Dunn, 1981, Levitin and Polyak, 1966) is the obvious linesearch extension of the steepest-descent method to deal with convex constraints, and is based on the iteration

$$
x_{k+1}=P_{\Omega}\left[x_{k}-\alpha_{k} \nabla_{x} f\left(x_{k}\right)\right]
$$

where $P_{\Omega}(v)$ projects $v$ into $\Omega$ and $\alpha_{k}$ is a suitable stepsize. In the case of simple bounds, $P_{\Omega}[v]=\operatorname{mid}(l, v, u)$, the (componentwise) median of $v$ with respect to the bounds $l$ and $u$, is trivial to compute. The method possesses one extremely helpful feature: for nondegenerate problems (i.e., those for which the removal of one or more active constraints necessarily changes the solution) the optimal "face" of active constraints will be determined in a finite number of iterations (Bertsekas, 1976). Of course, its steepest-descent ancestry hints that this is unlikely to be an effective method as it stands, and some form of acceleration is warranted. 
The simplest idea exploits the finite optimal-face identification property: if the active faces visited by consecutive iterates of the gradient-projection algorithm are identical, a higher order (Newton-like) method should be used to investigate this face. This was first suggested for quadratic $f$ (Moré and Toraldo, 1991), but is now commonplace for general objectives.

A natural question is whether there are other algorithms which have the finite optimalface identification property for non-degenerate problems. It turns out that the result is true for any algorithm for convex constraints for which the projected gradient $P_{T(x)}\left[-\nabla_{x} f(x)\right]$ converges to zero (Burke and Moré, 1988, Calamai and Moré, 1987)-here $T(x)$ is the closure of the cone of all feasible directions (the tangent cone) at $x$. Although the (discontinuous) projected gradient is often hard to compute, in the simple-bound case it is merely (componentwise)

$$
\left(P_{T(x)}\left[-\nabla_{x} f(x)\right]\right)_{i}=\left\{\begin{array}{cl}
-\min \left\{0,\left(\nabla_{x} f(x)\right)_{i}\right\} & \text { if } x_{i}=l_{i} \\
-\left(\nabla_{x} f(x)\right)_{i} & \text { if } l_{i}<x_{i}<u_{i} \text { and } \\
-\max \left\{0,\left(\nabla_{x} f(x)\right)_{i}\right\} & \text { if } x_{i}=u_{i} .
\end{array}\right.
$$

Its continuous variant $P_{\Omega}\left[x_{k}-\nabla_{x} f\left(x_{k}\right)\right]-x_{k}$ is sometimes preferred, and plays a similar role in theory and algorithms.

A restricted version of the finite identification result also holds in the degenerate case, namely that the set of strongly-active constraints (i.e., those whose removal will change the solution) will be identified in a finite number of iterations if the projected gradient converges to zero (Lescrenier, 1991, Burke and Moré, 1994). These finite-identification results apply to many contemporary methods.

Trust-region methods for the problem typically consider the gradient-projection arc

$$
d(\alpha)=P_{\Omega \cap\left\{y \mid\left\|y-x_{k}\right\| \leq \Delta_{k}\right\}}\left[x_{k}-\alpha \nabla_{x} f\left(x_{k}\right)\right]-x_{k},
$$

from $x_{k}$. Given a QN model $m_{k}(d)$, a so-called (generalized) Cauchy point $d\left(\alpha_{k}^{\mathrm{C}}\right)$ is found by approximately minimizing $m_{k}(d)$ along $d(\alpha)$; either the first local arc minimizer (Conn, Gould and Toint, 1988a) or a point satisfying sufficient-decrease linesearch conditions (Burke, Moré and Toraldo, 1990, Toint, 1988) is required - the computation of a suitable Cauchy point may be performed very efficiently when the Hessian is sparse (Conn, Gould and Toint, 1988b, Lin and Moré, 1999b). Thereafter a step $d_{k}$ is computed so that

$$
x_{k}+d_{k} \in \Omega, \quad\left\|d_{k}\right\| \leq \Delta_{k} \text { and } m_{k}\left(d_{k}\right) \leq m_{k}\left(d_{k}^{\mathrm{C}}\right)
$$

and the usual trust-region acceptance rules applied (e.g., Conn et al., 2000a, §6.1). Since it has been shown that the projected gradient converges to zero for these methods, the flexibility in (6) is typically used to accelerate convergence by allowing a truncated CG method to explore the face of active constraints at $x_{k}+d_{k}^{\mathrm{C}}$. Since the CG iterates may try to leave $\Omega$, early methods simply fixed variables to their bounds and restarted the $\mathrm{CG}$ iteration (Conn et al., 1988a), while more modern ones allow infeasible CG iterates by periodically projecting them back into $\Omega$ (Gould et al., 2003a, Lin and Moré, 1999b). 
If second derivatives are unavailable, they may be estimated by any of the methods discussed in $\S 2$. A particularly appealing approach is to use a limited-memory secant method to estimate the Hessian. Although this approximation is dense, it is so structured that a generalized Cauchy point may still be calculated. Moreover one of the advantages of limited memory methods, namely that the QN step may be computed directly, is retained, despite the requirement that the QN step be restricted to the face determined by $d_{k}^{\mathrm{C}}$, by judicious use of the Sherman-Morrison-Woodbury formula. (Byrd, Lu, Nocedal and Zhu, 1995).

Although we have only considered methods which remain feasible with respect to the bounds, there is no theoretical reason - so long as the objective function is well-defined outside $\Omega$-to do so provided there is some mechanism for ensuring that the iterates are asymptotically feasible (Facchinei, Lucidi and Palagi, 2002). It is also unsurprising that, just as in the unconstrained case, there is no need for the objective function to decrease monotonically so long as there is some overall monotonic trend (Facchinei et al., 1998, Gould et al., 2003a). Efforts have also been made to embed nonlinear CG methods within a gradient-projection framework (Pytlak, 1998). Filter ideas have also been investigated (Gould and Toint, 2003a) that use penalty techniques (see §5) to handle the bounds. Research is ongoing to merge filter methods with the projection methods discussed above or the interior-point techniques discussed below.

\subsubsection{Interior-point methods}

Interior-point methods provide an alternative means of solving bound-constrained problems. For simplicity, consider the case where $\Omega=\{x \mid x \geq 0\}$, suppose that $\mu$ is a positive - so called barrier - parameter, and let

$$
\phi(x, \mu) \stackrel{\text { def }}{=} f(x)-\mu \sum_{i=1}^{n} \log (x)_{i}
$$

be the logarithmic barrier function for the problem, where $(x)_{i}$ denotes the $i$-th component of $x$. The key idea is to trace approximate minimizers of $\phi(x, \mu)$ as $\mu$ decreases to zero. Under reasonable assumptions, and for sufficiently small positive values of $\mu$, (local) minimizers of $\phi(x, \mu)$ exist and describe continuous trajectories - primal central paths - converging to (local) solutions of the required problem (Fiacco and McCormick, 1968, Wright, 1992). Likewise, if $X$ is the diagonal matrix whose $i$-th diagonal element is the $i$-th component of $x$ and $e$ is a vector of ones, the associated (first-order) dual variables estimates

$$
z=\mu X^{-1} e,
$$

are located on trajectories enjoying similar properties and converge to Lagrange multipliers associated with the bound constraints. The cross product of each pair of trajectories is known as a primal-dual central path, and most barrier methods attempt to follow one with increasing accuracy as $\mu$ decreases (Fiacco and McCormick, 1968, Wright, 1992) -for this reason, interior-point methods are sometimes also referred to as path-following methods. 
The unconstrained minimization of $\phi$ can be handled using the techniques described in $\S 2$ so long as care is taken to ensure that the iterates remain within the interior of $\Omega$. A QN model of the form (2) might be used, and as such would be

$$
m_{k}(d)=\phi\left(x_{k}, \mu\right)+d^{T}\left(\nabla_{x} f\left(x_{k}\right)-\mu X_{k}^{-1} e\right)+\frac{1}{2} d^{T}\left(H_{k}+\mu X_{k}^{-2} e\right) d,
$$

where $H_{k}$ is, as before, an approximation to $\nabla_{x x} f\left(x_{k}\right)$. However, considerable numerical experience has shown that it is usually preferable to replace the first-order dual variable estimates $z_{k}=\mu X_{k}^{-1} e$ in the Hessian term of (8) to obtain instead

$$
m_{k}(d)=\phi\left(x_{k}, \mu\right)+d^{T}\left(\nabla_{x} f\left(x_{k}\right)-\mu X_{k}^{-1} e\right)+\frac{1}{2} d^{T}\left(H_{k}+X_{k}^{-1} Z_{k}\right) d,
$$

and to compute the dual variable $z_{k}$ by other means. In this case, since the optimal Lagrange multipliers for the problem are necessarily positive, it is reasonable to require the same be so of $z_{k}$. Rather than computing $z_{k}$ explicitly from (7), it is better to multiply both sides of (7) by $X$, giving $X z=\mu e$. Applying Newton's method to this last system then yields the alternative

$$
z_{k+1}=\mu X_{k}^{-1} e-X_{k}^{-1} Z_{k} d_{k}
$$

involving the current step $d_{k}$ from $x_{k}$. Additional safeguards need be employed to enforce convergence of the process (Conn et al., 2000a, Ch. 13). Methods of this latter type are referred to as primal-dual methods because they explicitly consider both primal and dual variables, while methods based on the model (8) (with its implicitly computed dual variables) are called primal methods.

An approximate minimizer of the model (9) may be computed by either a direct (factorization) or iterative (CG) method. If the latter is used, it is normally essential to precondition the iteration to remove the effects of the extreme eigenvalues of $X_{k}^{-1} Z_{k}$ (Luenberger, 1984, Ch. 12). A preconditioner of the form $P_{k}=G_{k}+X_{k}^{-1} Z_{k}$ for some suitable approximation $G_{k}$ of $H_{k}$ is usually recommended, with $G_{k}$ varying from naive $\left(G_{k}=0\right)$ to sophisticated $\left(G_{k}=H_{k}\right)$.

Both linesearch or trust-region globalization of interior-point methods are possible and essentially identical to that discussed in $\S 2$. The major difference in both cases is the addition of a so-called fraction-to-the-boundary rule, preventing iterates from prematurely approaching the boundary of the feasible set. A trust-region algorithm will accept a step $d_{k}$ from $x_{k}$ if

1. $x_{k}+d_{k} \geq \gamma_{k} x_{k}$ holds componentwise, for some given $0<\gamma_{k}<1$, and

2. there is good agreement between the changes in $m_{k}$ and $\phi(x, \mu)$.

For most practical purposes, the fraction-to-the-boundary parameter $\gamma_{k}$ is held constant, a typical value being 0.005. It may however be permitted to converge to zero, allowing for fast asymptotic convergence. Wächter and Biegler (2004) choose (in a more general context) $\gamma_{k}=\max \left(\gamma_{\min }, \mu_{k}\right)$, where $0<\gamma_{\min }<1$ is a prescribed minimal value. The fraction-to-the-boundary rule also applies for linesearch methods, and a (backtracking) 
linesearch is typically performed until it is satisfied. A corresponding backtracking may then also be applied to the dual step to ensure consistency.

Although there is no difficulty in providing a strictly-interior primal-dual starting point, $\left(x_{0}, z_{0}\right)$, in the bound-constrained case, it is generally sensible to ensure that such a point is well separated from the boundary of the feasible region; failure to do this can (and in practice does seriously) delay convergence. Given suitable primal estimates $x_{0}$, traditional choices for dual estimates $z_{0}$ include the vector of ones or those given from (7) although there is little reason to believe that these are more than heuristics. An initial value for $\mu$ is then typically $\mu_{0}=x_{0}^{T} z_{0} / n$, so as to obtain good centrality at the initial point.

It is well known that computing $d_{k}$ to be a critical point of (9) and recovering $z_{k+1}$ via (10) is equivalent to applying Newton's method from $\left(x_{k}, z_{k}\right)$ to the perturbed optimality conditions

$$
\nabla_{x} f(x)-z=0 \text { and } X z=\mu e
$$

for our problem. While it is tempting to try similar approaches directly with $\mu=0$ - socalled affine methods - these have both theoretical and practical shortcomings for general problems (see, for example, Conn et al., 2000a, §13.11). A more promising approach is to note that equivalent first-order optimality conditions are that

$$
W(x) \nabla_{x} f(x)=0
$$

where

$$
W(x)=\operatorname{diag} w(x) \text { and } w_{i}(x)=\left\{\begin{aligned}
x_{i} & \text { if }\left(\nabla_{x} f(x)\right)_{i} \geq 0 \\
-1 & \text { otherwise }
\end{aligned}\right.
$$

So long as strictly feasible iterates are generated, $W(x) \nabla_{x} f(x)$ is differentiable, and Newton's method may be applied to (11). To globalize such an iteration, combined linesearchtrust-region methods have been proposed (Coleman and Li, 1994 \& 1996) and variants which allow quadratic convergence even in the presence of degeneracy are possible (Heinkenschloss, Ulbrich and Ulbrich, 1999).

\subsubsection{Practicalities}

There have been a number of comparative studies of algorithms for bound-constrained optimization (Facchinei et al., 2002, Gould et al., 2003a, Lin and Moré, 1999b, Zhu, Byrd, Lu and Nocedal, 1997), but we feel that none of these makes a compelling case as to the best approach(es) for the large-scale case. In practice, both gradient projection and interior-point methods appear to require a modest number of iterations.

\subsubsection{Software}

Once again there is a reasonable choice of reliable software for the bound-constrained case. Both TRON (Lin and Moré, 1999b) and LANCELOT/SBMIN (Conn, Gould and Toint, 1992) are trust-region gradient-projection algorithms with subspace conjugate-gradient acceleration - there is an improved version of the latter within GALAHAD (Gould et al., 
2003a) - while L-BFGS-B (Zhu et al., 1997) is a line-search implementation of the limitedmemory approach. The Matlab function fmincon (Branch, Coleman and Li, 1999) uses an interior-point subspace method based on (11). FILTRANE, another algorithm of the GALAHAD library, uses a filter method combined with penalty techniques for the bounds. As before, more general codes such as KNITRO and LOQO are also highly appropriate.

\section{Large-scale linearly-constrained optimization}

As the next level of generality, we now turn to problems involving general linear constraints.

\subsection{Equality-constrained quadratic programming}

A - some might say the-basic subproblem in constrained optimization is to

$$
\underset{x \in \mathbb{R}^{n}}{\operatorname{minimize}} q(x)=g^{T} x+\frac{1}{2} x^{T} H x \text { subject to } A x=b,
$$

where $H$ is symmetric (but possibly indefinite) and $A$ is $m$ by $n$ and (without loss of generality) of full rank. Such equality-constrained quadratic programming (EQP) subproblems arise when computing search directions for either general methods for equality-constrained or active-set methods for inequality-constrained optimization. It is actually often more convenient to consider the related homogeneous problem

$$
\underset{\bar{x} \in \mathbb{R}^{n}}{\operatorname{minimize}} \bar{q}(\bar{x})=\bar{g}^{T} \bar{x}+\frac{1}{2} \bar{x}^{T} H \bar{x} \text { subject to } A \bar{x}=0 ;
$$

so long as there is some easy way to find $x_{0}$ satisfying $A x_{0}=b$, the solutions of the two problems satisfy $x=\bar{x}+x_{0}$ provided that $\bar{g}=g+H x_{0}$.

Critical points of (12) necessarily satisfy the augmented (KKT) system

$$
\left(\begin{array}{cc}
H & A^{T} \\
A & 0
\end{array}\right)\left(\begin{array}{l}
x \\
y
\end{array}\right)=\left(\begin{array}{c}
-g \\
b
\end{array}\right),
$$

and solutions of (14) can only be solutions of (12) if the coefficient matrix of (14) has precisely $m$ negative eigenvalues (Chabrillac and Crouzeix, 1984, Gould, 1985). Thus direct (factorization) methods for solving (14) must be capable of coping with indefinite matrices; fortunately there is a growing number of highly capable symmetric, indefinite linear solvers (for example BCSEXT, MA27/57, Oblio or PARDISO; see Scott, Hu and Gould, 2004, for a comparison), and in particular if $H$ is (block) diagonal a Schur-complement decomposition involving factorizations of $H$ and $-A H^{-1} A^{T}$ is often to be recommended. Nevertheless, for very large problems direct methods may be unviable or too expensive, and iterative methods may be the only alternative. Although non-symmetric or indefinite iterative methods may be applied, we only consider CG-type methods here, since these have the desirable property of decreasing $q(x)$ at every iteration. 
It should be apparent that CG methods can be applied explicitly to (13) by computing a basis $N$ for the null-space of $A$, and then using the transformation $\bar{x}=N x_{n}$ to derive the equivalent (unconstrained) problem of minimizing $q_{n}\left(x_{n}\right)=x_{n}^{T} g_{n}+\frac{1}{2} x_{n}^{T} H_{n} x_{n}$, where $g_{n}=N^{T} \bar{g}$ and $H_{n}=N^{T} H N$ are known as the reduced gradient and Hessian respectively. Perhaps not so obvious, the same may be achieved implicitly by using the standard preconditioned CG (PCG) method but using a block (so-called), constraint preconditioner of the form

$$
\left(\begin{array}{cc}
G & A^{T} \\
A & 0
\end{array}\right)\left(\begin{array}{c}
r \\
w
\end{array}\right)=-\left(\begin{array}{c}
\bar{g}+H x \\
0
\end{array}\right)
$$

to obtain the "preconditioned" residual $r$ from the "unpreconditioned" $\bar{g}+H x$, for some suitable $G$ (Coleman, 1994, Gould, Hribar and Nocedal, 2001, Lukšan and Vlček, 1998, Polyak, 1969). Various choices for $G$, ranging from the identity matrix to $H$, have been suggested, and all require a suitable (block) factorization of the coefficient matrix $K$ of (15); basic requirements are that $K$ should be non-singular and have precisely $m$ negative eigenvalues. A further advantage of the PCG approach is that any additional (properly-scaled) trust-region constraint may easily be incorporated using the GLTR strategy mentioned in $\S 2$. Nevertheless, requiring a factorization of $K$ may still be considered a disadvantage, and methods which avoid this are urgently needed.

\subsection{General quadratic programming}

Another important subproblem in constrained optimization is the general quadratic programming (QP) problem, namely to

$$
\underset{x \in \mathbb{R}^{n}}{\operatorname{minimize}} q(x) \text { subject to } A_{\mathcal{E}} x=b_{\mathcal{E}} \text { and } A_{\mathcal{I}} x \geq b_{\mathcal{I}}
$$

Of particular interest is the non-convex case where the symmetric $H$ may be indefinite, although in these circumstances we must normally be content with local solutions. The main application area we are concerned with is in solving subproblems which arise within sequential quadratic programming (SQP) algorithms for general nonlinear optimization (see $§ 5.1$, but note the caveat there that expresses our concerns over the SQP approach), although there are actually a large number of other (usually convex) applications of (16) (Gould, and Toint, 2000a), including VLSI design, optimal control, economic dispatch and financial planning, to mention only a few. They also constitute a class apart as their necessary and sufficient optimality conditions coincide (Contesse, 1980, Mangasarian, 1980, Borwein, 1982).

\subsubsection{Active-set methods for general quadratic programming}

As was the case for bound-constrained problems we considered in $\S 3$, QP methods may broadly be categorized as either active-set or interior-point based. As the name suggests, active-set methods aim to predict which of the inequality constraints $A_{\mathcal{I}} x \geq b_{\mathcal{I}}$ are active at a solution to (16). At each iteration, a working set $\mathcal{W}_{\mathcal{I}} \subseteq \mathcal{I}$ is selected so that the 
gradients of the constraints $A_{\mathcal{W}}, \mathcal{W}=\mathcal{E} \cup \mathcal{W}_{\mathcal{I}}$, are linearly independent. For this working set, the EQP

$$
\underset{x \in \mathbb{R}^{n}}{\operatorname{minimize}} q(x) \text { subject to } A_{\mathcal{W}} x=b_{\mathcal{W}}
$$

is solved (if possible) using one of the methods described in $§ 4.1$. There are a number of possibilities. If (17) is unbounded from below or if the solution to (17) violates one of the inequality constraints indexed by $\mathcal{I}-\mathcal{W}_{\mathcal{I}}$, one (or more) constraints should be added to the working set. If (17) is infeasible or if the solution to (17) is not that of (16) - the latter is true if any of the Lagrange multipliers $y$ from (14) are negative - one (or more) constraints should be removed from the working set. In the convex case, only when the solution to (17) satisfies all of the constraints indexed by $\mathcal{I} \backslash \mathcal{W}_{\mathcal{I}}$ and all of the Lagrange multipliers are positive can we be certain that we have solved (16). Unfortunately, for non-convex problems, even checking if such a critical point is a local minimizer may be (NP) hard (Murty and Kabadi, 1987). While such a strategy is simple - it may be reduced to the solution of a sequence of EQPs - potentially a large number of iterations may be required. Fortunately, just as with the simplex method for linear programming (LP), the working set usually changes very gradually, and the potentially dominant cost of matrix factorization is lessened through updates to existing factors. Thus active-set methods for QP usually comprise a large number of very cheap iterations - by contrast interior-point methods require a few, more expensive ones.

There have been relatively few active-set methods for large-scale QP, especially in the nonconvex case. The majority of these are based on the idea of inertia control (Fletcher, 1971). Suppose that the coefficient matrix $K$ for the optimality conditions (14) corresponding to the current EQP (17) has the "correct" inertia, i.e., $K$ has $|\mathcal{W}|$ negative eigenvalues. If a constraint is added to the working set, the new subproblem will inherit the correct inertia. However, if a constraint is removed from the working set, it is possible that the resulting $K$ may have $|\mathcal{W}|+1$ rather than $|\mathcal{W}|$ negative eigenvalues. If this happens, there must be a feasible direction of negative curvature, and an inertia-controlling method will follow this direction until it encounters a currently inactive constraint (or perhaps $q$ is unbounded from below on the feasible set). This new constraint will be added to the working set, and once again the resulting $K$ will have either $|\mathcal{W}|$ or $|\mathcal{W}|+1$ negative eigenvalues. In the former case, the correct inertia has been restored, while in the latter there is again a direction of feasible negative curvature. This process of following negative curvature and adding currently inactive constraints must ultimately terminate (unless the problem is unbounded below) at a vertex of the feasible region at which point the correct inertia will have been restored. The principal differences between the inertia-controlling methods that have been proposed are the algebraic means by which the factors are maintained and updated. These include using Schur-complement (Gill, Murray, Saunders and Wright, 1990, Gill, Murray, Saunders and Wright, 1991) or linear-programming basis-type (Gould, 1991) updates to a factorization of an initial $K$, or Cholesky-factor updates of the (dense) reduced Hessian (Fletcher, 2000), the latter only really being appropriate for problems with few degrees of freedom. 
For problems for which a direct solution of the sequence of generated EQPs is unviable or too expensive, it is also possible to use the PCG method described in $\S 4$.1. Now rather than controlling the inertia of the KKT matrix, inertia control is only required for the preconditioner (15). Once again, factors of the preconditioner must adapt to changes in the working set, but the ability to choose $G$ gives considerable flexibility (Gould and Toint, 2002a).

\subsubsection{Interior-point methods for general quadratic programming}

It is easy to generalize the interior-point methods discussed in $\S 3.0 .3$ to cope with the quadratic program (16). Denoting the $i$-th row of $A_{\mathcal{I}}$ by $a_{i}$ and the $i$-th component of $b_{\mathcal{I}}$ by $b_{i}$, typical barrier methods for such problems aim to

$$
\begin{aligned}
& \underset{x \in \mathbb{R}^{n}}{\operatorname{minimize}} \phi(x, \mu) \stackrel{\text { def }}{=} q(x)-\mu \sum_{i \in \mathcal{I}} \log \left(a_{i}^{T} x-b_{i}\right) \\
& \text { subject to } A_{\mathcal{E}} x=b_{\mathcal{E}}
\end{aligned}
$$

as $\mu$ decreases to 0 , while ensuring that $x$ remains interior to $\Omega_{\mathcal{I}}=\left\{x \mid A_{\mathcal{I}} x \geq b_{\mathcal{I}}\right\}$. Just as in the bound-constrained case and under reasonable conditions, the minimizers of (18) and their dual variable (Lagrange multiplier) estimates

$$
y_{\mathcal{I}}=\mu C_{\mathcal{I}}^{-1}(x) e, \text { where } C_{\mathcal{I}}(x)=\operatorname{diag} c_{\mathcal{I}}(x) \text { and } c_{\mathcal{I}}(x)=A_{\mathcal{I}} x-b_{\mathcal{I}},
$$

define continuous trajectories - primal-dual central paths - leading to (local) solutions of (16).

For fixed $\mu$ and feasible $x_{k}$, basic iterative methods might compute a suitable step $d_{k}$ by building a primal QN model

$$
m_{k}(d)=\phi\left(x_{k}, \mu\right)+d^{T}\left(g_{k}-\mu A_{\mathcal{I}}^{T} C_{\mathcal{I} k}^{-1} e\right)+\frac{1}{2} d^{T}\left(H+\mu A_{\mathcal{I}}^{T} C_{\mathcal{I} k}^{-2} A_{\mathcal{I}}\right) d
$$

where $g_{k} \stackrel{\text { def }}{=} H x_{k}+g$ and $C_{\mathcal{I} k} \stackrel{\text { def }}{=} C_{\mathcal{I}}\left(x_{k}\right)$, and then trying to (approximately)

$$
\underset{s \in \mathbb{R}^{n}}{\operatorname{minimize}} m_{k}(d) \text { subject to } A_{\mathcal{E}} s=0 \text { and (possibly) }\|s\| \leq \Delta_{k},
$$

involving an additional trust-region constraint. To ensure feasibility of the next iterate, a stepsize $0<\alpha_{k} \leq \alpha_{k}^{\max }$ should be imposed along an approximate solution $d_{k}$ to (20)-a fraction-to-the-boundary rule such as

$$
\alpha_{k}^{\max }=\max \left\{0<\alpha \leq 1 \mid c_{\mathcal{I}}\left(x_{k}+\alpha d_{k}\right) \geq \gamma_{k} c_{\mathcal{I}}\left(x_{k}\right)\right\}
$$

is appropriate - and to guarantee convergence it may also be necessary to linesearch along $d_{k}$ or adjust $\Delta_{k}$ in the usual manner. But as in $\S 3.0 .3$, a primal-dual model

$$
m_{k}(d)=\phi\left(x_{k}, \mu\right)+d^{T}\left(g_{k}-\mu A_{\mathcal{I}}^{T} C_{\mathcal{I} k}^{-1} e\right)+\frac{1}{2} d^{T}\left(H+A_{\mathcal{I}}^{T} C_{\mathcal{I} k}^{-1} Y_{\mathcal{I} k} A_{\mathcal{I}}\right) d
$$


involving explicit (positive) dual variables $y_{\mathcal{I} k}$ is generally preferable to (19) both in theory and in practice (Conn, Gould, Orban and Toint, 2000b). In particular, the analog of the Newton update (10),

$$
y_{\mathcal{I} k+1}=\mu C_{\mathcal{I} k}^{-1} e-C_{\mathcal{I} k}^{-1} Y_{\mathcal{I} k} d_{k}
$$

is appropriate, so long as appropriate precautions are taken to modify (22) to ensure that $y_{\mathcal{I} k+1}$ remains sufficiently positive (Conn et al., $2000 a$, Ch. 13).

The key subproblem here is (20), but this is precisely of the form discussed in $§ 4.1$. The only significant extra issue when the objective function has the form (21) is that any preconditioner should respect the potentially ill-conditioned Hessian term (Luenberger, 1984, Ch. 12), and thus that the leading block in (15) should be $G_{k}+A_{\mathcal{I}}^{T} C_{\mathcal{I} k}^{-1} Y_{\mathcal{I} k} A_{\mathcal{I}}$ for some suitable approximation $G_{k}$ to $H$. Although it might first appear that such a leading block may be unacceptably dense, the preconditioning step (15) for the model (21) may be trivially rearranged to give the potentially sparser

$$
\left(\begin{array}{ccc}
G_{k} & A_{\mathcal{E}}^{T} & A_{\mathcal{I}}^{T} \\
A_{\mathcal{E}} & 0 & 0 \\
A_{\mathcal{I}} & 0 & -Y_{\mathcal{I} k}^{-1} C_{\mathcal{I} k}
\end{array}\right)\left(\begin{array}{c}
r \\
w_{\mathcal{E}} \\
w_{\mathcal{I}}
\end{array}\right)=-\left(\begin{array}{c}
g_{k}+H s \\
0 \\
-\mu Z_{\mathcal{I} k}^{-1} e
\end{array}\right)
$$

for auxiliary variables $w_{\mathcal{I}}=C_{\mathcal{I} k}^{-1} Z_{\mathcal{I} k} A_{\mathcal{I}} s-\mu C_{\mathcal{I} k}^{-1} e$ (Gould, 1986).

The method sketched above presupposes that an initial point $x_{0}$ is known within the intersection of $\Omega_{\mathcal{E}}=\left\{x \mid A_{\mathcal{E}} x=b_{\mathcal{E}}\right\}$ and the interior of $\Omega_{\mathcal{I}}$. A suitable point may be found by solving an auxiliary phase-I problem such as

$$
\underset{x \in \mathbb{R}^{n}, s_{\mathcal{I}} \in \mathbb{R}^{n_{\mathcal{I}}}}{\operatorname{minimize}}-\sum_{i \in \mathcal{I}} \log \left(s_{i}\right) \text { subject to } A_{\mathcal{E}} x=b_{\mathcal{E}} \text { and } A_{\mathcal{I}} x-s_{\mathcal{I}}=b_{\mathcal{I}},
$$

where the $s_{\mathcal{I}}$ are being treated as auxiliary, positive slack variables. The intention here is to find a point which is significantly interior, and in the above case will give the analytic center of the feasible region. Fortunately, although finding feasible points for (24) may not be obvious, the problem is convex and may be solved using an infeasible interior-point method, such as those discussed in the next section.

With this in mind, an equivalent formulation of (16) is to

$$
\underset{x \in \mathbb{R}^{n}, s_{\mathcal{I}} \in \mathbb{R}^{n_{\mathcal{I}}}}{\operatorname{minimize}} q(x) \text { subject to } A_{\mathcal{E}} x=b_{\mathcal{E}}, \quad A_{\mathcal{I}} x-s_{\mathcal{I}}=b_{\mathcal{I}} \text { and } s_{\mathcal{I}} \geq 0
$$

and an alternative barrier method for (16) might aim to

$$
\begin{aligned}
& \underset{x \in \mathbb{R}^{n}, s_{\mathcal{I}} \in \mathbb{R}^{n_{\mathcal{I}}}}{\operatorname{minimize}} \phi\left(x, s_{\mathcal{I}}, \mu\right) \stackrel{\text { def }}{=} q(x)-\mu \sum_{i \in \mathcal{I}} \log \left(s_{i}\right) \\
& \text { subject to } A_{\mathcal{E}} x=b_{\mathcal{E}} \text { and } A_{\mathcal{I}} x-s_{\mathcal{I}}=b_{\mathcal{I}}
\end{aligned}
$$

as $\mu$ decreases to 0 . Although the distinction between this "slack variable" formulation and (18) is actually very slight if the constraints $A_{\mathcal{I}} x-s_{\mathcal{I}}=b_{\mathcal{I}}$ are enforced throughout, as we shall see later the distinction is more pronounced for nonlinear constraints. 


\subsubsection{Interior-point methods for convex quadratic programming}

Quadratic programs are traditionally classified as convex or nonconvex, depending on whether the Hessian matrix $H$ is positive semidefinite or not. Simply finding a local minimizer of a nonconvex QP is an NP-hard problem (Vavasis, 1990), as is proving that a first-order critical point is in fact a minimizer (Murty and Kabadi, 1987) - most algorithms for general QP are consequently only designed to locate first-order critical points.

Convex QPs are provably solvable by algorithms having polynomial complexity (Nesterov and Nemirovskii, 1994, Vavasis, 1991). The use of a barrier function to force convergence in the convex case is usually inefficient, and the best methods are more closely allied to those for LP (Wright, 1997). The basis of these primal-dual path-following methods for convex QP is to solve the perturbed optimality conditions

$$
\begin{aligned}
g+H x-A_{\mathcal{E}}^{T} y_{\mathcal{E}}-A_{\mathcal{I}}^{T} y_{\mathcal{I}} & =0 \\
A_{\mathcal{E}} x-b_{\mathcal{E}} & =0, \\
\text { and } Y_{\mathcal{I}}\left(A_{\mathcal{I}} x-b_{\mathcal{I}}\right)-\mu e & =0
\end{aligned}
$$

for (16) or, more commonly,

$$
\begin{aligned}
g+H x-A_{\mathcal{E}}^{T} y_{\mathcal{E}}-A_{\mathcal{I}}^{T} y_{\mathcal{I}} & =0, \\
y_{\mathcal{I}}-z_{\mathcal{I}} & =0, \\
A_{\mathcal{E}} x-b_{\mathcal{E}} & =0, \\
A_{\mathcal{I}} x-s_{\mathcal{I}}-b_{\mathcal{I}} & =0, \\
\text { and } Z_{\mathcal{I}} s_{\mathcal{I}}-\mu e & =0
\end{aligned}
$$

for (25), using Newton's method or a variant thereof, while maintaining strict feasibility for $s_{\mathcal{I}} \geq 0$ and $z_{\mathcal{I}} \geq 0$ (or $A_{\mathcal{I}} x \geq b_{\mathcal{I}}$ and $y_{\mathcal{I}}$ for (16)), and letting $\mu$ gradually decrease to zero. The most popular are based on the linesearch-based predictor-corrector algorithm of Mehrotra (1992), originally developed for LP.

A typical predictor-corrector iteration for (28) involves the solution of a pair of (symmetrized) linear systems of the form

$$
\left(\begin{array}{ccccc}
G & 0 & A_{\mathcal{E}}^{T} & A_{\mathcal{I}}^{T} & 0 \\
0 & 0 & 0 & -I & -I \\
A_{\mathcal{E}} & 0 & 0 & 0 & 0 \\
A_{\mathcal{I}} & -I & 0 & 0 & 0 \\
0 & -I & 0 & 0 & -Z_{\mathcal{I}}^{-1} S_{\mathcal{I}}
\end{array}\right)\left(\begin{array}{c}
d_{x} \\
d_{s_{\mathcal{I}}} \\
-d_{y_{\mathcal{E}}} \\
-d_{y_{\mathcal{I}}} \\
d_{z_{\mathcal{I}}}
\end{array}\right)=-\left(\begin{array}{c}
g(x)-A_{\mathcal{E}}^{T} y_{\mathcal{E}}-A_{\mathcal{I}}^{T} y_{\mathcal{I}} \\
y_{\mathcal{I}}-z_{\mathcal{I}} \\
c_{\mathcal{E}}(x) \\
c_{\mathcal{I}}(x)-s_{\mathcal{I}} \\
-Z_{\mathcal{I}}^{-1} r_{\mathrm{C}}(\mu)
\end{array}\right)
$$

for different values of $r_{\mathrm{C}}(\mu)$, where (as before) $c_{\mathcal{I}}(x)=A_{\mathcal{I}} x-b_{\mathcal{I}}, c_{\mathcal{E}}(x)=A_{\mathcal{E}} x-b_{\mathcal{E}}$, $g(x)=g+H x$ and $G \approx H$. Note that Newton's method for (28) results when $G=H$ and $r_{\mathrm{C}}(\mu)=S_{\mathcal{I}} z_{\mathcal{I}}-\mu e$ with the current value of $\mu$. The first of the two systems uses $r_{\mathrm{C}}(\mu)=S_{\mathcal{I}} z_{\mathcal{I}}$ and defines a predictor step intended to reduce primal and dual feasibility. This step is often referred to as an affine scaling step and is denoted $d^{\mathrm{AFF}}$. A steplength $\alpha^{\mathrm{AFF}}$ is determined to preserve positivity of $z_{\mathcal{I}}$ and $s_{\mathcal{I}}$. Upon defining the duality gap after the predictor step $\mu^{\mathrm{AFF}}=\left(z_{\mathcal{I}}+\alpha^{\mathrm{AFF}} d_{z_{\mathcal{I}}}^{\mathrm{AFF}}\right)^{T}\left(s_{\mathcal{I}}+\alpha^{\mathrm{AFF}} d_{s_{\mathcal{I}}}^{\mathrm{AFF}}\right)$ and the centering parameter 
$\sigma=\left(\mu^{\mathrm{AFF}} / \mu\right)^{\tau}$ with $2 \leq \tau \leq 4$, the second system uses $r_{\mathrm{C}}(\mu)=S_{\mathcal{I}} z_{\mathcal{I}}-\sigma \mu e+D_{s_{\mathcal{I}}}^{\mathrm{AFF}} d_{z_{\mathcal{I}}}^{\mathrm{AFF}}$ and defines a corrector step aiming to improve centrality. The final primal and dual common steplength (Mehrotra, 1992) is determined from

$$
\alpha=\min \left(1, \eta \alpha_{\mathrm{MAX}}^{\mathrm{P}}, \eta \alpha_{\mathrm{MAX}}^{\mathrm{D}}\right),
$$

where $\eta \in[0.9,1.0)$ converges to 1 as a solution is approached, and $\alpha_{\mathrm{MAx}}^{\mathrm{P}}$ and $\alpha_{\mathrm{MAx}}^{\mathrm{D}}$ are primal and dual steplengths enforcing a fraction-to-the-boundary rule.

The higher-order corrections scheme of Gondzio (1996), again originally developed for LP, generalizes to convex quadratic programming. Several corrector-like steps are taken, as long as substantial steplengths are acceptable and individual complementarity pairs cluster around their average value. These steps aim for dynamically-computed targets (Jansen, Roos, Terlaky and Vial, 1996) located in a loose neighbourhood of the central path. The number of corrector steps is computed at the first iteration by balancing the cost of the linear algebra and the expected progress towards optimality.

Just as in $\S 4.1$, (block) direct or iterative methods may be used to solve the indefinite system (29). Further savings often result from the block elimination

$$
\left(\begin{array}{ccc}
G & A_{\mathcal{E}}^{T} & A_{\mathcal{I}}^{T} \\
A_{\mathcal{E}} & 0 & 0 \\
A_{\mathcal{I}} & 0 & -Z_{\mathcal{I}}^{-1} S_{\mathcal{I}}
\end{array}\right)\left(\begin{array}{c}
d_{x} \\
-y_{\mathcal{E}}-d_{y_{\mathcal{E}}} \\
-y_{\mathcal{I}}-d_{y_{\mathcal{I}}}
\end{array}\right)=-\left(\begin{array}{c}
g(x) \\
c_{\mathcal{E}}(x) \\
c_{\mathcal{I}}(x)-Z_{\mathcal{I}}^{-1} r_{\mathrm{C}}(\mu)
\end{array}\right)
$$

of (29), or possibly even from

$$
\begin{array}{r}
\left(\begin{array}{cc}
G+A_{\mathcal{I}}^{T} S_{\mathcal{I}}^{-1} Z_{\mathcal{I}} A_{\mathcal{I}} & A_{\mathcal{E}}^{T} \\
A_{\mathcal{E}} & 0
\end{array}\right)\left(\begin{array}{c}
d_{x} \\
-y_{\mathcal{E}}-d_{y_{\mathcal{E}}}
\end{array}\right)=- \\
\left(\begin{array}{c}
g(x)+A_{\mathcal{I}}^{T} S_{\mathcal{I}}^{-1} Z_{\mathcal{I}}\left[c_{\mathcal{I}}(x)-Z_{\mathcal{I}}^{-1} r_{\mathrm{C}}(\mu)\right] \\
c_{\mathcal{E}}(x)
\end{array}\right)
\end{array}
$$

which arises by further eliminating $y_{\mathcal{I}}+d_{y_{\mathcal{I}}}$ from (30) - of course (31) may be inappropriate if the term $A_{\mathcal{I}}^{T} S_{\mathcal{I}}^{-1} Z_{\mathcal{I}} A_{\mathcal{I}}$ is significantly denser than $G$, but has the virtue of being considerably smaller if there are many inequality constraints. It is also worth noting that the corresponding predictor-corrector steps for (27) satisfy

$$
\left(\begin{array}{ccc}
G & A_{\mathcal{E}}^{T} & A_{\mathcal{I}}^{T} \\
A_{\mathcal{E}} & 0 & 0 \\
A_{\mathcal{I}} & 0 & -Y_{\mathcal{I}}^{-1} C_{\mathcal{I}}
\end{array}\right)\left(\begin{array}{c}
d_{x} \\
-y_{\mathcal{E}}-d_{y_{\mathcal{E}}} \\
-y_{\mathcal{I}}-d_{y_{\mathcal{I}}}
\end{array}\right)=-\left(\begin{array}{c}
g(x) \\
c_{\mathcal{E}}(x) \\
c_{\mathcal{I}}(x)-Y_{\mathcal{I}}^{-1} r_{\mathrm{C}}(\mu)
\end{array}\right)
$$

which is simply $(30)$ in the special case $s_{\mathcal{I}}=c_{\mathcal{I}}(x)$ and $z_{\mathcal{I}}=y_{\mathcal{I}}$-also c.f. (23). The coefficient matrices from $(29),(30) /(32)$ and (31) are appropriate preconditioners for PCG so long as they have, respectively, $\operatorname{rank}\left(A_{\mathcal{E}}\right)+2|\mathcal{I}| \operatorname{rank}\left(A_{\mathcal{E}}\right)+|\mathcal{I}|$ and $\operatorname{rank}\left(A_{\mathcal{E}}\right)$ negative eigenvalues (Conn et al., 2000b, with Sylvester's law of inertia); equivalently $G+A_{\mathcal{I}}^{T} S_{\mathcal{I}}^{-1} Z_{\mathcal{I}} A_{\mathcal{I}}$ should be positive definite on the null-space of $A_{\mathcal{E}}$, and this will always be the case if $G$ is positive definite. Any of the factorizations mentioned in (4.1) are appropriate. 
For large problems, it is vital to be able to exploit commonly-occurring sub-structure when solving (30). Applications from multi-stage stochastic programming, network communications or asset liability management give rise to matrices $H$ and $A$ having one of a number of predefined block structure examples include $H$ and $A$ being block diagonal, primal or dual block angular or bordered block diagonal. Moreover, this block structure appears recursively in the sense that the structure of the blocks is similar to that of the matrix containing them. This nestedness is fully exploitable on observing that if matrices $H$ and $A$ have compatible structures - i.e., have the same number of diagonal blocks with matching numbers of columns - the coefficient matrix $K$ of (30) can be reordered to have similarly exploitable block structure (Gondzio and Grothey, 2003a).

Frequently in practice $K$ may be very ill-conditioned or even singular, and it is common to regularize $K$ to avoid such deficiencies. Typically diagonal blocks $R$ will be added to $K$ so that the resulting matrix $K+R$ is quasi-definite. Quasi-definite matrices (Vanderbei, 1995) are strongly factorizable in the sense that, for any symmetric permutation $P$, there exist a unit lower triangular matrix $L$ and a diagonal matrix $D$ such that $P(K+R) P^{T}=L D L^{T}$ without recourse to $2 \times 2$ pivoting, as is common with other popular factorizations of indefinite matrices (see $\S 4.1$ ). If the system is block-structured as above, the quasi-definite factorization may easily be parallelized, since block structure in $P(K+R) P^{T}$ induces block structure in $L$ and $D$ (Gondzio and Grothey, 2003a).

The requirement that slack variables introduced in (25) remain strictly feasible suggests that a steplength $0<\alpha_{k} \leq \alpha_{k}^{\max }$ be chosen where

$$
\alpha_{k}^{\max }=\max \left\{0<\alpha \leq 1 \mid s_{\mathcal{I}, k}+\alpha d_{s_{\mathcal{I}}} \geq \gamma_{k} s_{\mathcal{I}, k}\right\}
$$

to enforce a fraction-to-the-boundary rule. A similar rule applies to primal variables that are subject to bounds and to dual variables. A strictly feasible initial point is any $s_{\mathcal{I}, 0}>0$, but in practice it may be prudent to initialize $s_{\mathcal{I}}$ to a significantly positive value. Since the inequality constraints also need to be satisfied, a common choice is to pick $s_{\mathcal{I}, 0}=$ $\max \left(A_{\mathcal{I}} x_{0}-b_{\mathcal{I}}, \sigma e\right)$ componentwise, where $x_{0}$ is supplied by the user or the model, $\sigma>0$ is a given constant, e.g., $\sigma=1$ and $e$ is the vector of all ones. Often, explicit bound constraints will be honoured by first moving $x_{0}$ to satisfy them, and computing $s_{\mathcal{I}, 0}$ from this perturbed initial point. Another possibility is to compute an affine-scaling step $d^{\mathrm{AFF}}$, i.e., using $\mu=0$, for the primal-dual system associated with (16). Upon defining $s_{\mathcal{I}}^{\mathrm{AFF}}=s_{\mathcal{I}, 0}+d_{s_{\mathcal{I}}}^{\mathrm{AFF}}$, an initial $s_{\mathcal{I}, 1}$ is computed based on the feasibility of $s_{\mathcal{I}}^{\mathrm{AFF}}$, using a rule such as

$$
s_{\mathcal{I}, 1}=\max \left(\beta e,\left|s_{\mathcal{I}}^{\mathrm{AFF}}\right|\right) \quad \text { or } \quad s_{\mathcal{I}, 1}=s_{\mathcal{I}}^{\mathrm{AFF}}+\gamma \max \left(0,-s_{\mathcal{I}}^{\mathrm{AFF}}\right)+\beta e,
$$

where the absolute values and maxima are understood elementwise and $\beta, \gamma>0$ (Gertz, Nocedal and Sartenaer, 2003).

Good general-purpose initial values for the Lagrange multipliers $y$ in primal-dual interior methods are hard to find, and poor guesses may introduce unwarranted nonconvexity into the model if the problem is nonconvex. Nonetheless, they are often initialized to approximate least-squares solutions for dual feasibility, i.e., values of $y$ for which the gradient 
lies on the nullspace of the constraints at the starting point, and adjusted to ensure that those corresponding to inequality constraints are strictly positive.

Not all path-following interior-point methods for convex QP are of the predictorcorrector type. The simplest alternative is to solve (29) or (30) for a pre-assigned $\mu$ but to ensure ensure strict feasibility by means of a fraction-to-the-boundary rule, in which the stepsize $\alpha$ is chosen as

$$
\alpha=\min \left\{1,(1-\epsilon) \max _{\left[d_{s}\right]_{i}<0} \frac{s_{i}}{-\left[d_{s}\right]_{i}},(1-\epsilon) \max _{\left[d_{z}\right]_{i}<0} \frac{z_{i}}{-\left[d_{z}\right]_{i}}\right\},
$$

for a small $\epsilon>0$. A merit function such as

$$
\phi\left(x, s_{\mathcal{I}}, y, z_{\mathcal{I}}\right) \equiv s_{\mathcal{I}}^{T} z_{\mathcal{I}}+\left\|\nabla \mathcal{L}\left(x, s_{\mathcal{I}}, y_{\mathcal{E}}, y_{\mathcal{I}}, z_{\mathcal{I}}\right)\right\|_{2}
$$

where $\mathcal{L}\left(x, s_{\mathcal{I}}, y_{\mathcal{E}}, y_{\mathcal{I}}, z_{\mathcal{I}}\right)$ is the Lagrangian associated with $(25)$, is used to assess suitability of such a steplength. Such a fraction-to-the-boundary condition may be implicitly ensured by Zhang's (1994) stepsize rule, and in this case yields a global linear convergence rate and a polynomial algorithm.

One further interesting idea in both convex and non-convex cases is to solve (16) by a sequence of minimizations over the intersection of the interior of the feasible region with iteratively-generated low-dimensional (typically 2- or 3-dimensional) subspaces. The advantage here is that the resulting subproblems are small, so that global optimization is possible. Clearly the choice of subspaces is crucial, and should include at least one "descent" direction for whatever globalization mechanism is to be used, and others which are geared towards fast asymptotic convergence - solutions of (29) for different $r_{\mathrm{C}}(\mu)$ may be used (Boggs, Domich, Rogers and Witzgall, 1996).

\subsubsection{Practicalities}

The only comparison of the competing QP ideologies we are aware of is that of Gould and Toint (2002b). As perhaps one might expect, the interior-point approach seems generally to be preferable to the active-set especially for very large problems where the number of active-set iterations can be enormous. For "warm-start" problems where a solution to a small perturbation of an existing already solved problem is required, there is some virtue in using the active-set approach as it seems better able to use good estimates of the optimal active set. Whether this trend will continue is debatable, especially as current research for LP indicates promise for warm-started interior-point methods (Gondzio and Grothey, 2003b, Yildirim and Wright, 2002).

When carefully implemented, interior methods for QP scale almost perfectly with the number of variables, and rarely do they need more than, say, 30-35 iterations. Moreover, unlike active-set-type methods, the linear systems which arise at each iteration have identical block structure. Nonetheless, the solution of such systems may still be costly, and implementations must pay particular attention to exploiting structure - an example of a 
disastrous situation caused by the lack of exploitation of low-rank-corrector structure is given by Ferris and Munson (2000).

A final important idea is to simplify QPs before solution. Such "presolve" methods have proved to be very effective for LP (Gondzio, 1997), and similar gains are also possible for QP (Gould and Toint, 2004c).

\subsubsection{Software}

Currently available active-set non-convex QP codes include VE09 (Gould, 1991), bqpd (Fletcher, 2000) and QPA (Gould and Toint, 2002a). The PRESOLVE package (Gould and Toint, 2004c) is, as its name suggests, intended for presolving QPs.

Highly efficient commercial interior-point-based software such as CPLEX 6.0 (1998), MOSEK (Andersen and Andersen, 2000) and XPRESS-MP (Guéret, Prins and Seveaux, 2002 ) is available for convex QP. These packages implement path-following algorithms in a primal-dual setting, and are available for parallel machines as well as for personal computers. Significantly, they may be tested online on the NEOS Server for Optimization (Czyzyk, Mesnier and Moré, 1998, Gropp and Moré, 1997, Dolan, 2001).

The object-oriented QP package OOQP (Gertz and Wright, 2003) implements generalizations of both Mehrotra's (1992) predictor-corrector and Gondzio's (1996) higher-order correction methods. OOQP has the advantage of being customizable to various application domains, and has been tailored to solve problems arising from support vector machines and Huber regression. Similar features are implemented in COPLQP (Ye, 1997).

Specialized structure is exploited automatically by the object-oriented parallel solver OOPS (Gondzio and Grothey, 2003a). Currently OOPS has been able to solve non-trivial problems involving 52 million variables and 20 million constraints.

Although now a code for general nonlinear programming, a set of default parameter values for convex QP and a careful implementation of a tailored $L D L^{T}$ factorization for the quasidefinite systems at the heart of the algorithm make LOQO (Vanderbei, 1999) one of the most robust predictor-corrector, primal-dual path-following convex QP solvers. Much of this is due to the care with which the factorization is obtained. An $L D L^{T}$ factorization of the regularized matrix $K+R$ from (30) is computed using a two-stage ordering scheme assigning priorities to pivots based on estimates of the fill-in in both $A A^{T}$ and $A^{T} A$. Pivots corresponding to the current priority are treated using a minimum-degree ordering heuristic.

QPB from the GALAHAD library of Gould et al. (2003a) implements a primal-dual interior method for general QP — for non-convex problems, QPB is only capable of identifying a weak second-order critical point. The Phase-I relies on the package LSQP, itself a primal-dual infeasible method for convex separable QP (Zhang, 1994) which is also part of GALAHAD. Numerical tests on a monoprocessor machine on small, $n+m \lesssim 10^{4}$, medium, $10^{4} \lesssim n+m \lesssim 10^{5}$ and large-scale, $10^{5} \lesssim n+m \lesssim 10^{6}$, problems illustrate how well the method scales with the dimension, and the superiority of interior-point approaches over active-set type methods when a reliable estimate of the optimal working set is not available 
and when the number of variables and constraints are large (Conn et al., 2000b, Gould et al., 2003a, Gould and Toint, 2002b).

\subsection{General linearly-constrained optimization}

When the constraints are linear but the objective neither linear nor quadratic, most algorithms try to emulate the QP methods described above, by ensuring feasibility with respect to constraints and requiring a reduction in the objective function (or perhaps barrier function) at each iteration - if an interior-point method is used, the iterates will remain interior to all inequality constraints. The only significant differences occur because the Hessian of the objective function changes at each iteration and must be periodically evaluated or estimated by some means. If the objective is close to linear, solutions (and intermediate iterates) often have a high proportion of active constraints $(|W| \approx n)$ and some methods (Murtagh and Saunders, 1982, Gill, Murray and Saunders, 2002, Friedlander and Saunders, 2005) exploit this by maintaining (dense) secant approximations of the reduced Hessian.

Interior-point methods for convex problems have received extensive attention since the existence of self-concordant barriers lead to polynomial algorithms (Nesterov and Nemirovskii, 1994, Renegar, 2001), and specialized methods have been devised for important applications. A good example is the minimization of a nonlinear but convex, (and preferably, but not necessarily) separable objective subject to linear equalities and bounds which arise in transportation planning, knowledge management or world-wide web traffic modeling (Saunders and Tomlin, 1996). The problem is stated as

$$
\underset{x \in \mathbb{R}^{n}}{\operatorname{minimize}} f(x) \text { subject to } A x=b \text { and } l \leq x \leq u,
$$

and is regularized and reformulated as

$$
\underset{x, r}{\operatorname{minimize}} f(x)+\frac{1}{2}\left\|D_{1} x\right\|^{2}+\frac{1}{2}\|r\|^{2} \text { subject to } A x+D_{2} r=b \text { and } l \leq x \leq u,
$$

for some diagonal positive-definite regularization matrices $D_{1}$ and $D_{2}$. A primal-dual pathfollowing method is then applied. The bulk of the computation involves solving systems of the form

$$
\left(\begin{array}{cc}
H & A^{T} \\
A & -D_{2}^{2}
\end{array}\right)\left(\begin{array}{c}
d_{x} \\
-d_{y}
\end{array}\right)=-\left(\begin{array}{c}
\nabla_{x} f(x)-A^{T} y-\mu\left[(X-L)^{-1}+(U-X)^{-1}\right] e \\
A x+D_{2}^{2} y-b
\end{array}\right)
$$

where $H=\nabla_{x x} f(x)+D_{1}^{2}+(X-L)^{-1} Z_{l}+(U-X)^{-1} Z_{u}$ and $y, z_{l}$ and $z_{u}$ are suitable Lagrange multiplier estimates. As the coefficient matrix here is quasi-definite, it admits an $L D L^{T}$ factorization. Alternatively, eliminating $d_{y}$ to obtain normal equations and treating them as a least-squares problem, a trial step $\left(d_{x}, d_{y}\right)$ is computed using a least-squares method, e.g., LSQR of Paige and Saunders (1982).

Not all proposed interior-point methods are of the path following variety. For example it is possible to generalize the affine-scaling approach of Coleman and $\mathrm{Li}$ (1996) to handle linear inequality constraints (Coleman and Li, 2000). 


\subsubsection{Software}

Although it is capable of handling general constraints, the venerable active-set NLP solver MINOS (Murtagh and Saunders, 1982) is perhaps best regarded for its ability to deal with linear constraints. Likewise its successors SNOPT (Gill et al., 2002) and KNOSSOS (Friedlander and Saunders, 2005) are both highly effective for such problems, particularly if there are relatively few degrees of freedom. As usual, other general nonlinear programming packages, such as LOQO and KNITRO may be applied and are comfortable with such problems, although we would not recommend LANCELOT in this case.

\section{Large-scale nonlinearly-constrained optimization}

Finally, we turn our attention to our most general nonlinear programming problem (1) and the attendant difficulties of coping with constraint curvature.

\subsection{Sequential linear and quadratic programming methods}

The phrase "sequential quadratic programming" (SQP) seems to mean different things to different people, but the central theme is undoubtedly to apply an iteration for which a new iterate is generated by trying to minimize a quadratic approximation of the appropriate Lagrangian function $\ell(x, y) \stackrel{\text { def }}{=} f(x)-y_{\mathcal{E}}^{T} c_{\mathcal{E}}(x)-y_{\mathcal{I}}^{T} c_{\mathcal{I}}(x)$ subject to linearizations of some or all of the constraints. Here we will examine several aspects of this approach. There have been a number of surveys of SQP methods over the past 10 years (Boggs and Tolle, 1995 \& 2000, Conn, Gould and Toint, 1997, Gould and Toint, 2000) and we urge readers to consult these for details since we do not have room to give them all here.

We start by considering problems only involving equality constraints - for some people, for example those who work on PDE-constrained optimization (e.g., Biros and Ghattas, 2000), this is SQP - for which the central ideas are best understood. But it is in the context of the general problem (1) which we believe that most people understand the term SQP, and which we consider next. There is a strong distinction between linearizing a subset of the constraints at each iteration - the EQP subproblem approach, which is strongly influenced by methods for equality constraints - and linearizing all constraints at every iteration - the IQP subproblem approach.

\subsection{SQP methods for equality-constrained problems}

We first consider SQP methods for the equality-constrained (EC) problem

$$
\underset{x \in \mathbb{R}^{n}}{\operatorname{minimize}} f(x) \text { subject to } c_{\mathcal{E}}(x)=0 .
$$


SQP methods for EC problems (SQPE) aim to find a correction $d_{k}$ to the current solution estimate $x_{k}$ so as to (approximately)

$$
\begin{aligned}
& \underset{d}{\operatorname{minimize}} q(d)=d^{T} g\left(x_{k}\right)+\frac{1}{2} d^{T} H_{k} d \\
& \text { subject to } c_{\mathcal{E}}(x)+J_{\mathcal{E}}\left(x_{k}\right) d=0 .
\end{aligned}
$$

Here $g(x)=\nabla_{x} f(x)$ is the gradient of the objective, $J(x)=\nabla_{x} c_{\mathcal{E}}(x)$ is the Jacobian of the constraints, and $H_{k}$ is (an approximation to) the Hessian of the Lagrangian function $\ell_{\mathcal{E}}\left(x, y_{\mathcal{E}}\right)=f(x)-y_{\mathcal{E}}^{T} c_{\mathcal{E}}(x)$ for given estimates $y_{\mathcal{E} k}$ of the Lagrange multipliers $y_{\mathcal{E}}$ at $x_{k}$. If $H_{k}=\nabla_{x x} \ell_{\mathcal{E}}\left(x_{k}, y_{\mathcal{E} k}\right)$ and

$$
d^{T} H_{k} d>0 \text { for all } d \text { for which } J_{\mathcal{E}}\left(x_{k}\right) d=0,
$$

the solution to (36) is identical to that obtained by applying Newton's method to the criticality conditions $\nabla_{\left(x, y_{\mathcal{E}}\right)} \ell_{\mathcal{E}}\left(x, y_{\mathcal{E}}\right)=0$ at $\left(x_{k}, y_{\mathcal{E} k}\right)$. Aside from the fundamental issues of how to choose $H_{k}$ and $y_{\mathcal{E} k}$, SQPE methods have a number of obvious possible shortcomings. In particular (i) the linearized constraints may be inconsistent, (ii) (37) may be violated, and (iii) the iteration may diverge.

Possible shortcoming (i) is best dealt with in one of two, related, ways. The first is to re-pose (1) as the related penalty problem

$$
\underset{x \in \mathbb{R}^{n}}{\operatorname{minimize}} \phi(x, \sigma) \stackrel{\text { def }}{=} f(x)+\sigma\left\|c_{\mathcal{E}}(x)\right\|,+\sigma \sum_{i \in \mathcal{E}}\left|c_{i}(x)\right|+\sigma \sum_{i \in \mathcal{I}} \min \left(-c_{i}(x), 0\right)
$$

for some sufficiently large $\sigma>0$ and given norm $\|\cdot\|$, and instead to minimize some model of the (non-smooth) penalty function $\phi(x, \sigma)$. A typical model problem then might be to approximately

$$
\underset{d}{\operatorname{minimize}} q(d)+\sigma\left\|c_{\mathcal{E}}(x)+J_{\mathcal{E}}\left(x_{k}\right) d\right\|
$$

if $\|\cdot\|$ is polyhedral (e.g., the $\ell_{1}$ - or $\ell_{\infty}$-norm), (39) may be reformulated as a (consistent) inequality-constrained QP, while if $\|\cdot\|$ is elliptical (e.g., the $\ell_{2}$-norm) a quadratic conicprogramming reformulation is possible.

Notice that the intention here is implicitly to allow inconsistent linearized constraints by merely reducing their infeasibility as much as is possible. A second, more direct way of dealing with inconsistency is to aim for reduction in infeasibility rather than full satisfaction of the constraints. A composite step $d_{k}=n_{k}+t_{k}$ may be used to achieve this. The idea is simply that the "(quasi-)normal" step $n_{k}$ tries to reduce $\left\|c_{\mathcal{E}}(x)+J_{\mathcal{E}}\left(x_{k}\right) n\right\|$ while the "tangential" step $t_{k}$ aims to reduce $q(d)$ while maintaining the infeasibility at the level achieved by $n_{k}$-if $n_{k}$ reduces the infeasibility to zero and $t_{k}$ solves

$$
\underset{t \in \mathbb{R}^{n}}{\operatorname{minimize}} q\left(n_{k}+t\right) \text { subject to } J_{\mathcal{E}}\left(x_{k}\right) t=0,
$$

$d_{k}$ will be the solution to (36). Although there are a number of composite-step methods (Conn et al., 2000a, §15.4), the most appealing is the so-called Byrd-Omojokun approach 
(Byrd, Hribar and Nocedal, 1999, Lalee, Nocedal and Plantenga, 1998, Omojokun, 1989) in which the $\mathrm{CG}$ method is used both to reduce $\left\|c_{\mathcal{E}}(x)+J_{\mathcal{E}}\left(x_{k}\right) n\right\|_{2}^{2}$ and subsequently to approximately solve the EQP (40) (see $§ 4.1)$.

If shortcoming (ii) occurs, $q(d)$ will be unbounded from below on the feasible region. Suitable remedies are just as in the unconstrained case (see §2). Linesearch-based methods cope with such an eventuality by either obtaining a direction of feasible negative curvature or by modifying $H_{k}$, although good methods for achieving the latter during matrix factorization are still in their infancy (Forsgren, 2002, Forsgren and Murray, 1993, Gould, 1999). Trust-region-based methods impose a constraint to stop steps to infinity, but there is the added complication that the trust-region constraint $\|s\| \leq \Delta_{k}$ may be incompatible with the linearizations $J_{\mathcal{E}}\left(x_{k}\right) d=-c_{\mathcal{E}}(x)$ if $\Delta_{k}$ is too small. In this case, one of the remedies proposed for shortcoming (i) may be required.

Shortcoming (iii) may be overcome in the usual way, namely by requiring descent (monotonic or otherwise) with respect to a suitable merit function such as (38). A good choice of $\sigma$ is vital if such a method is to be efficient, and we will return to this later. An unfortunate consequence - the Maratos (1978) "effect" - is that the SQP step may not be acceptable to merit functions like (38), and that an auxiliary calculation (a "second-order correction") may be required to modify the step to allow fast convergence. Other merit functions, such as the augmented Lagrangian function, avoid this defect and have been used with much success (Boggs, Kearsley and Tolle, 1999a, Gill et al., 2002).

A modern alternative to merit functions, which avoids the need to compute a penalty parameter, is to use the filter idea introduced in $\S 2.1 .3$. For EC problems, we consider the conflicting objectives $\theta_{1}(x)$ and $\theta_{2}(x)$ to be the objective function and the constraints violation $\left\|c_{\mathcal{E}}(x)\right\|$, respectively. A step $d$ is thus accepted if either the objective function decreases or if the constraints violation is reduced, while it is rejected if no decrease is obtained in either. But of course many further refinements are necessary in order to devise a workable algorithm. One is the way that filter methods deal with incompatible model constraints. Rather than resorting to the remedies for shortcoming (i) given above, filter trust-region algorithms switch to a "restoration phase", i.e. to the minimization of the constraint violation alone (the objective function is momentarily forgotten) until a model with compatible constraints is found. Since this will be true for any feasible point for the original problem, this restoration phase must terminate at a suitable point so long as it is capable of finding, or indeed possible to find, one. This restoration phase may use any suitable algorithm, including the filter method for nonlinear least-squares mentioned in $\S 2.2$. It may also be triggered more frequently - the method of Gonzaga, Karas and Vanti (2003) performs the equivalent of a restoration phase at every iteration.

A drawback that is common to SQPE approaches is that they all potentially suffer from the Maratos effect and therefore may need a second-order correction step to guarantee fast convergence. In theory this may be avoided by the filter remembering Lagrangian rather than objective function values (Ulbrich, 2004b), but, to our knowledge, numerical experience is not yet available to support this idea in practice. 
Rival trust-region SQPE filter methods impose different requirements on the step computation-Fletcher, Leyffer and Toint (2002b) require the global solution of the trustregion constrained SQPE, while others (Fletcher and Leyffer, 2002, Fletcher, Gould, Leyffer, Toint and Wächter, 2002a, Gonzaga et al., 2003, Gould and Toint, 2004a, 2003b) permit approximate local minimizers - and on the precise technique for maintaining the filter. This technical decision is often based on the distinction between iterations whose main effect is to reduce the objective function ( $f$-iterations), and iterations whose main effect is to reduce constraint violation ( $\theta$-iterations).

Linesearch variants of the filter idea are also possible. Despite using a different globalization technique, the proposal of Wächter and Biegler (2003a, 2003b) remains similar in structure to the trust-region variants, in that it also involves restorations, second-order correction steps and similarly uses the distinction between $f$-and $\theta$-iterations to manage the filter.

For all SQPE algorithms, two other issues which are of great practical importance are the choice of Hessian approximation $H_{k}$ and Lagrange multiplier estimates $y_{\mathcal{E} k}$. Although exact second derivatives of the Hessian of the Lagrangian are often available, the use of approximations still persists especially for problems where $|\mathcal{E}| \approx n$. In particular, as we noted in $\S 4$, solving (40) may be reduced to minimizing $t_{n}^{T} g_{n}+\frac{1}{2} t_{n}^{T} H_{n} t_{n}$ and recovering $t_{k}=N^{T} t_{n}$, where $g_{n}=N^{T}\left(g\left(x_{k}\right)+H_{k} n_{k}\right)$ and $H_{n}=N^{T} H_{k} N$, and the columns of $N$ form a basis for the null-space of $J_{\mathcal{E}}\left(x_{k}\right)$. Thus so long as $|\mathcal{E}| \approx n, H_{n}$ will be small and it will be feasible to maintain $H_{n}$ as a dense secant approximation to $N^{T} \nabla_{x x} \ell_{\mathcal{E}}\left(x_{k}, y_{\mathcal{E} k}\right) N$ (see the survey articles mentioned at the start of this section). If $|\mathcal{E}| \not \approx n$, it may still be possible to maintain a useful limited-memory secant approximation to the same matrix (Gill et al., 2002). Lagrange multipliers $y_{\mathcal{E} k+1}$ are often taken as those from the approximate solution to (38), although some form of interpolation between these values and $y_{\mathcal{E} k}$ may be necessary if the merit function, the trust-region or constraint inconsistency intervene; little work seems to have been performed to discover the influence of such distractions which is somewhat surprising given the influence $y_{\mathcal{E} k}$ may have on $H_{k}$. As an alternative, a direct or CG least-squares solution to $J_{\mathcal{E}}^{T}\left(x_{k}\right) y=g\left(x_{k}\right)$ may be appropriate (Lalee et al., 1998).

\subsection{SQP methods for the general problem}

Suffice to say, as the epithet suggests, an SQP method aims to solve the general problem (1) by solving a sequence of (cleverly) chosen QP problems. There are essentially two classes of SQP methods.

\subsubsection{Sequential equality-constrained quadratic programming (SEQP) meth- ods}

The first, which we call sequential equality-constrained QP (SEQP) methods are essentially $\mathrm{SQPE}$ methods for which the set $\mathcal{E}$ is replaced by a (changing) estimate $\mathcal{A}_{k} \subseteq \mathcal{E} \cup \mathcal{I}$ of (1)'s optimal active set. All of the salient points we made about SQPE methods apply equally 
here, but now the dynamic data structures necessary to accommodate changes in $\mathcal{A}_{k}$ and, more importantly, the choice of $\mathcal{A}_{k}$ itself introduce extra complications. Of paramount importance is the globalization strategy, since otherwise there will be little control over constraints not in $\mathcal{A}_{k}$. In particular, it is vital that all constraints are represented in whatever merit function or filter is used.

A common strategy is to use the non-differentiable penalty function

$$
\underset{x \in \mathbb{R}^{n}}{\operatorname{minimize}} \phi(x, \sigma) \stackrel{\text { def }}{=} f(x)+\sigma\left\|c_{\mathcal{E}}(x)\right\|+\sigma\left\|\min \left(-c_{\mathcal{I}}(x), 0\right)\right\|
$$

as a merit function, and to use an EQP model in which a second-order approximation to the (locally) differentiable part of $\phi(x, \sigma)$ is minimized subject to linearized approximations to the (locally) non-differentiable part remaining fixed (Coleman and Conn, 1982); $\mathcal{A}_{k}$ is thus defined by those constraints with (almost) zero values.

An alternative is to use the active set at a minimizer of a "simpler" model of (1) or (41) to predict the active set of (1). The most obvious models are linear, and lead to linear programming suproblems which aim to

$$
\begin{gathered}
\underset{d}{\operatorname{minimize}} l(d)=d^{T} g\left(x_{k}\right) \\
\text { subject to } c_{\mathcal{E}}(x)+J_{\mathcal{E}}\left(x_{k}\right) d=0 \\
\text { and } c_{\mathcal{I}}(x)+J_{\mathcal{I}}\left(x_{k}\right) d \geq 0
\end{gathered}
$$

or

$$
\underset{d}{\operatorname{minimize}} l(d)+\sigma\left\|c_{\mathcal{E}}(x)+J_{\mathcal{E}}\left(x_{k}\right) d\right\|+\sigma\left\|\min \left(-c_{\mathcal{I}}(x)-J_{\mathcal{I}}\left(x_{k}\right) d, 0\right)\right\| ;
$$

the advantage here is that there are excellent (simplex and interior-point) methods for large-scale linear programming. However since the solutions to these subproblems almost inevitably lie at vertices of their feasible regions, and as there is no reason to expect that the solution to (1) has $n$ active constraints, (42) or (43) alone are not sufficient to determine $\mathcal{A}_{k}$.

One way of remedying this is to impose artificial constraints whose role is simply to cut off those problem constraints which are likely to be inactive at the solution to (1); if an artificial constraint is active at the solution of (42) or (43) it will not be included in $\mathcal{A}_{k}$. Care must be taken, however, to ensure that the artificial constraints do not exclude optimally active problem constraints, and the balance between these aims is quite delicate. Early sequential linear programming (SLP) methods (Griffith and Stewart, 1961) imposed artificial constraints of the form $\|s\|_{\infty} \leq \Delta$ in which $\Delta$ was dynamically adjusted, but it was Fletcher and Sainz de la Maza (1989) who first interpreted this as a trust-region constraint. Crucially they showed that the usual trust-region acceptance and adjustment rules are sufficient to correctly identify the optimal active set in a finite number of iterations. Both filter- (Chin and Fletcher, 2003) and merit-function- (Fletcher and Sainz de la Maza, 1989, Byrd et al., 2004a) based SLP variants are possible.

If the non-differentiable penalty function $\phi(x, \sigma)$ in $(41)$ is used, it is important that the penalty parameter $\sigma$ be adjusted to ensure that ultimately feasible critical points of the latter correspond to critical points of (1). Although in principle one could simply 
adjust $\sigma$ once an approximate solution of (41) has been found (Mayne and Polak, 1976), this is wasteful. It is preferable to adjust $\sigma$ as soon as there is model-based evidence that the current value is not reducing the constraints, and means for doing this while ensuring convergence to critical points of (1) (or perhaps finding a critical point of infeasibility) are known (Byrd, Gould, Nocedal and Waltz, 2004b).

\subsubsection{Sequential inequality-constrained quadratic programming (SIQP) meth- ods}

The second class of SQP methods are those we refer to as sequential inequality-constrained QP (SIQP) methods. In these, no a priori prediction is made about the active set, but instead a correction $d_{k}$ is chosen to (approximately)

$$
\begin{gathered}
\underset{d}{\operatorname{minimize}} q(d) \text { subject to } c_{\mathcal{E}}(x)+J_{\mathcal{E}}\left(x_{k}\right) d=0 \\
\text { and } c_{\mathcal{I}}(x)+J_{\mathcal{I}}\left(x_{k}\right) d \geq 0
\end{gathered}
$$

Now $H_{k}$ is an approximation to the Hessian of the full Lagrangian, $\ell(x, y)=f(x)-y^{T} c(x)$, for the problem, and linearizations of all constraints are included. Although we no longer need to specify $\mathcal{A}_{k}$, constraint inconsistency and iterate divergence are still serious concerns, and now we have the added complication that (iv) (44) may have (many) local minimizers.

Given all of these potential pitfalls, why are SIQP methods so popular? One reason is obviously their potential for fast local convergence; under reasonable assumption, the iteration based on (44) will correctly identify the active set and thereafter converge rapidly (Robinson, 1974). Another is favourable empirical evidence accumulated on small-scale problems (Hock and Schittkowski, 1981). But, on this basis and given the growing number of successful codes for large-scale QP, it might be thought surprising that there are so few large-scale SIQP algorithms. We now believe that this is not a coincidence and most likely an indication of the unsuitability of the SIQP paradigm for large-scale optimization. Why do we believe this?

Our first objection to SIQP is simply that given even the most efficient QP method, the cost of solving a large-scale inequality-constrained QP (IQP) is usually far greater than, say, an equivalently-sized EQP or interior-point subproblem. Thus a method that uses IQPs needs either to ensure that relatively few overall iterations are required, or have some mechanism for stopping short of QP optimality. Although there is anecdotal evidence that SIQP methods require few iterations for small-scale problems, we are unaware of any proof that this will always be the case. Likewise, the methods suggested in the tiny body of work on IQP truncation (Goldsmith, 1999, Murray and Prieto, 1995) may, in the worst case, require the solution of $n$ (related) EQPs per IQP.

Of more serious concern are the dangers posed by allowing indefinite $H_{k}$. This possibility rarely surfaced in the small-scale case, since almost always positive-definite secant Hessian approximations were used. But for large problems traditional secant approximations are rarely viable on sparsity grounds - limited-memory secant methods are possible 
(Gill et al., 2002) but may give inaccurate approximations, while the alternatives of using partitioned secant approximations or exact second derivatives often generate indefinite Hessians. Indefinite $H_{k}$ may cause difficulties for a number of reasons. Firstly, the possibility of moving to an unwelcome (possibly higher) local minimizer, $d_{k}$, cannot be discounted, particularly when using an interior-point QP solver. Such a $d_{k}$ may well be unsuitable for use with a globalisation strategy. While this appears to be a defect specifically for interiorpoint QP solvers, active-set methods may also be fooled. Consider the simple-bound QP

$$
\underset{x \in \mathbb{R}^{2}}{\operatorname{minimize}} \frac{1}{2}\left(x_{1}^{2}+x_{2}^{2}\right)-3 x_{1} x_{2}-\frac{5}{4} x_{1}+\frac{7}{4} x_{2} \text { subject to } 0 \leq x_{1}, x_{2} \leq 1
$$

whose contours are illustrated in Figure 1-this is a simplified version of that given by Goldsmith (1999). Starting from $x=(0,0)$, many active-set QP solvers would move

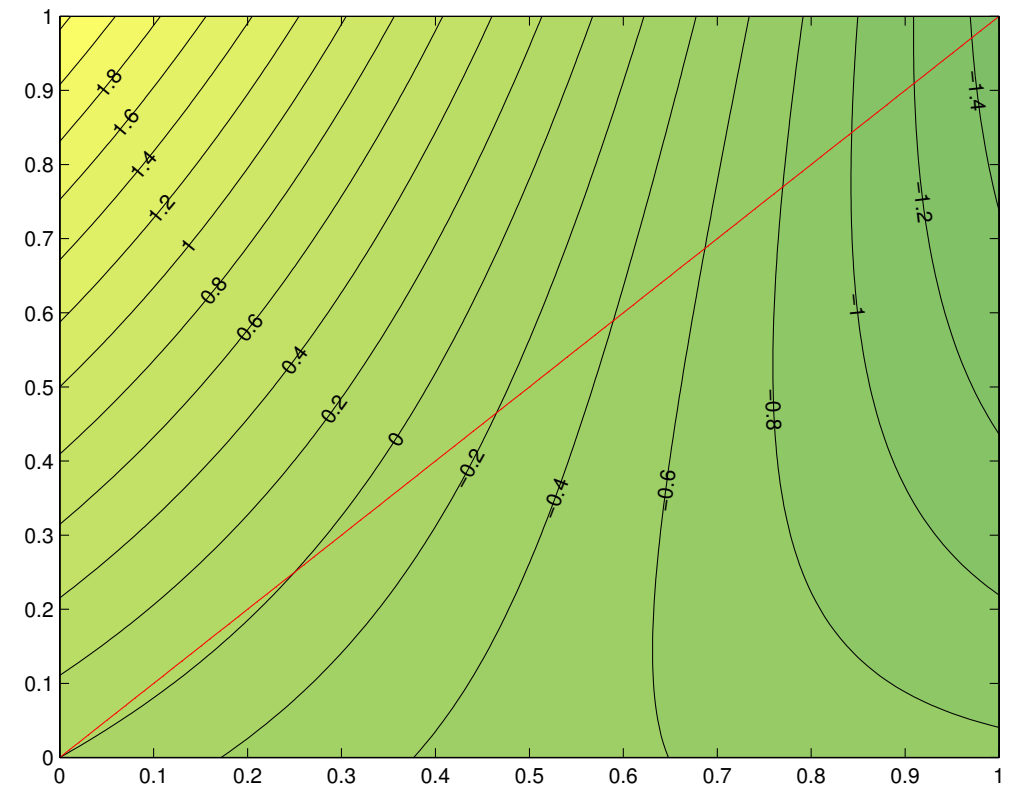

Figure 1: Active-set QP method gives uphill step

downhill via the corner $(1,0)$ to the (global) minimizer at $(1,1)$ - both steps are along directions of positive curvature. Unfortunately the overall step $(1,1)$ is an initially-uphill direction of negative curvature, and thus again unlikely to be suitable for use with a globalisation strategy. Of course this does not mean that the method will fail, merely that the approach may be inefficient as extra precautions (such as reducing a trust-region radius or modifying curvature) may have to be applied.

Whatever our reservations, SIQP methods remain popular. Linesearch, trust-region and filter variants have been proposed. Some avoid difficulty (iv) above by insisting on positive-definite (sometimes limited-memory) secant approximations to second derivatives (Gill et al., 2002). Others modify true second derivatives to ensure that the reduced Hessian is positive definite (Boggs, Kearsley and Tolle, 1999b, Boggs et al., 1999a), while 
some use the restoration-phase of the filter approach to recover from bad steps (Fletcher and Leyffer, 2002).

\subsection{Interior-point methods for nonlinear programs}

As the reader might anticipate, the last decade has seen an explosion in interest in pathfollowing methods for the general nonlinear program (1). Amongst the large number of papers devoted to the topic, two related approaches have emerged.

The first places all inequality constraints directly into a logarithmic barrier, leaving only explicit equality constraints. A sequence of barrier subproblems of the form

$$
\begin{aligned}
& \underset{x \in \mathbb{R}^{n}}{\operatorname{minimize}} \phi(x ; \mu) \stackrel{\text { def }}{=} f(x)-\mu \sum_{i \in \mathcal{I}} \log \left(c_{i}(x)\right) \\
& \text { subject to } c_{\mathcal{E}}(x)=0
\end{aligned}
$$

parametrized by the scalar $\mu>0$, is solved for positive values of $\mu$ which eventually decrease to zero. This approach is particularly appealing when $c_{\mathcal{E}}(x)=0$ are sufficiently simple to be handled directly, e.g. when they are linear (Conn et al., 2000b) -indeed, this is simply a generalization of (18) - but does require that the inequality constraints are strictly satisfied throughout. Since this may be difficult to achieve - even finding an initial point for which this is true may be far from trivial - the second approach allows inequality constraints to be violated at intermediate stages, but for each introduces a slack variable which is treated by a barrier function. The resulting problem is thus of the form

$$
\begin{aligned}
& \underset{x \in \mathbb{R}^{n}, s_{\mathcal{I}} \in \mathbb{R}^{n_{\mathcal{I}}}}{\operatorname{minimize}} \phi(x, s ; \mu) \stackrel{\text { def }}{=} f(x)-\mu \sum_{i \in \mathcal{I}} \log \left(s_{i}\right) \\
& \text { subject to } c_{\mathcal{E}}(x)=0 \text { and } c_{\mathcal{I}}(x)-s_{\mathcal{I}}=0 .
\end{aligned}
$$

Clearly, the introduction of slacks $s_{\mathcal{I}}$ is reminiscent of (26). Although it is vital that the slacks remain strictly feasible throughout, not all methods of this type remain infeasible right up to the solution (Byrd, Nocedal and Waltz, 2003).

For both approaches, the barrier subproblems are equality constrained, and the SQPE methods described in $\S 5.2$ are appropriate (Byrd, Gilbert and Nocedal, 2000a, Vanderbei and Shanno, 1999, Wächter and Biegler, 2004). Note however that extra precautions to ensure that the barrier terms remain finite must be taken, and it is here that (46) has some advantage, since in this case the barrier terms only involve (trivial) linear expressions.

Just as in the linearly-constrained case, locally convergent methods may be devised by applying (variants of) Newton's method to the perturbed optimality conditions

$$
\begin{aligned}
\nabla f(x)+J_{\mathcal{E}}(x)^{T} y_{\mathcal{E}}-J_{\mathcal{I}}(x)^{T} y_{\mathcal{I}} & =0 \\
c_{\mathcal{E}}(x) & =0 \\
\text { and } c_{\mathcal{I}}(x) y_{\mathcal{I}}-\mu e & =0
\end{aligned}
$$


of (1), or

$$
\begin{aligned}
\nabla f(x)+J_{\mathcal{E}}(x)^{T} y_{\mathcal{E}}-J_{\mathcal{I}}(x)^{T} y_{\mathcal{I}} & =0 \\
y_{\mathcal{I}}-z_{\mathcal{I}} & =0 \\
c_{\mathcal{E}}(x) & =0 \\
c_{\mathcal{I}}(x)-s_{\mathcal{I}} & =0 \\
\text { and } Z_{\mathcal{I}} s_{\mathcal{I}}-\mu e & =0
\end{aligned}
$$

of

$$
\underset{x \in \mathbb{R}^{n}, s_{\mathcal{I}} \in \mathbb{R}^{n_{\mathcal{I}}}}{\operatorname{minimize}} f(x) \text { subject to } c_{\mathcal{E}}(x)=0, c_{\mathcal{I}}(x)-s_{\mathcal{I}}=0 \text { and } s_{\mathcal{I}} \geq 0,
$$

(c.f. (27) and (28)). The only differences between the variants on Newton's method described in $\S 4.2 .3$ and those applicable here are that the Jacobians $A_{\mathcal{E}}$ and $A_{\mathcal{I}}$ in (29)(32) are now $J_{\mathcal{E}}(x)$ and $J_{\mathcal{I}}(x)$, respectively, and that $G$ should now be an approximation to the Hessian of the Lagrangian, $\nabla_{x x} \ell(x, y)$; (direct or iterative) methods for solving these systems are identical to those in $§ 4.2 .3$.

In (46), when the constraint Jacobian

$$
J(x)=\left(\begin{array}{ll}
J_{\mathcal{E}}(x) & 0 \\
J_{\mathcal{I}}(x) & I
\end{array}\right)
$$

has full rank, composite step (reduced space) variants of (29)-(32) are also possible. Just as in $§ 5.2$, the step may be decomposed using the Byrd-Omojokun scheme (Omojokun, 1989), and least-squares estimates of the Lagrange multipliers for the equality constraints obtained. If similar multiplier estimates for inequality constraints are found, care needs to be taken to ensure that these remain positive (Wright, 1997) or that the quadratic model remains convex in the slacks (Byrd et al., 1999). For problems arising from, e.g., dynamical systems where multiplier estimates are not available, it is remarkable that schemes to update the penalty parameter may still be derived (Wächter, 2002).

As always, it is necessary to globalize Newton's method in some way, and both (smooth and non-smooth) merit function- and filter-based possibilities have been proposed. Issues that arise with the linesearch globalization of the Newton direction $d_{x}$

$$
\left(\begin{array}{ccc}
G & J_{\mathcal{E}}^{T}(x) & J_{\mathcal{I}}^{T}(x) \\
J_{\mathcal{E}}(x) & 0 & 0 \\
J_{\mathcal{I}}(x) & 0 & -Z_{\mathcal{I}}^{-1} S_{\mathcal{I}}
\end{array}\right)\left(\begin{array}{c}
d_{x} \\
-y_{\mathcal{E}}-d_{y_{\mathcal{E}}} \\
-y_{\mathcal{I}}-d_{y_{\mathcal{I}}}
\end{array}\right)=-\left(\begin{array}{c}
g(x) \\
c_{\mathcal{E}}(x) \\
c_{\mathcal{I}}(x)-s_{\mathcal{I}}+\mu Z_{\mathcal{I}}^{-1} e
\end{array}\right)
$$

(or its $§ 4.2 .3$ equivalents (29)-(32)) include the choices of stepsize and other (penalty and barrier) parameters and strategies to ensure that $G$ is chosen to guarantee that $d_{x}$ gives descent for whatever merit function is used - to date, the simple expedient of adding a diagonal matrix $\lambda I$ to $G$ for suitably large $\lambda$ seems to be the most sophisticated strategy used in the large-scale case (Vanderbei and Shanno, 1999, Wächter and Biegler, 2004), although, just as in $\S 4.2 .3$, all that is actually required is that $G+J_{\mathcal{I}}^{T}(x) S_{\mathcal{I}}^{-1} Z_{\mathcal{I}} J_{\mathcal{I}}(x)$ should be positive definite on the null-space of $J_{\mathcal{E}}(x)$. Murray and Wright (1992) devised a linesearch procedure tailored to the logarithmic barrier function, given a search direction 
$d$, by identifying the closest constraint for which $d$ is a descent direction at the current iterate. A stepsize is computed by identifying a root of an approximation to the gradient of the barrier along $d$, by linearizing $f$ and the constraint in question and ignoring all other constraints. Several other interpolating functions are used as approximations of the logarithmic barrier by the same authors, so as to devise specialized linesearches. To the best of our knowledge, these have not been incorporated into large-scale interior-point codes.

Typical merit functions for (46) might be the non-smooth penalty-barrier function (Yamashita, Yabe and Tanabe, 2004)

$$
\phi(x, s ; \mu, \nu)=f(x)-\mu \sum_{i \in \mathcal{I}} \log \left(s_{i}\right)+\nu\left\|c_{\mathcal{E}}(x)\right\|+\nu\left\|c_{\mathcal{I}}(x)-s_{\mathcal{I}}\right\|
$$

the smooth variant (Gay, Overton and Wright, 1998, Vanderbei and Shanno, 1999)

$$
\psi(x, s ; \mu, \nu)=f(x)-\mu \sum_{i \in \mathcal{I}} \log \left(s_{i}\right)+\frac{\nu}{2}\left\|c_{\mathcal{E}}(x)\right\|_{2}^{2}+\frac{\nu}{2}\left\|c_{\mathcal{I}}(x)-s_{\mathcal{I}}\right\|_{2}^{2},
$$

or some scaled equivalents, perhaps even involving a different penalty parameter $\nu_{i}$ per constraint to account for poor scaling. Although these parameters should be handled globally as described in $§ 5.3 .1$, care must be taken to ensure that the direction computed from (50) or its variants is a descent direction for the merit function. This may be guaranteed for $\phi$ by iteratively increasing the penalty parameter until its directional derivative is negative; only a finite number of increases are required under standard assumptions (Byrd, Gilbert and Nocedal, 2000b).

A disadvantage of (51) and (52) is that they really only measure suitability of the primal step $d_{x}$; other means are used to compute steps in the dual variables. One function which does not suffer from this drawback is the augmented penalty-barrier merit function (Forsgren and Gill, 1998),

$$
\begin{aligned}
\theta(x, y ; \mu, \nu)=f(x) & +\frac{1}{2 \mu} \sum_{i \in \mathcal{E}}\left\{c_{i}(x)^{2}+\nu\left(c_{i}(x)+\mu y_{i}\right)^{2}\right\} \\
& -\mu \sum_{i \in \mathcal{I}}\left\{\log \left(c_{i}(x)\right)+\nu\left(\log \left(\frac{c_{i}(x) y_{i}}{\mu}\right)+1-\frac{c_{i}(x) y_{i}}{\mu}\right)\right\},
\end{aligned}
$$

which allows simultaneous minimization in both the primal and dual variables. So long as $G+J_{\mathcal{I}}^{T}(x) S_{\mathcal{I}}^{-1} Z_{\mathcal{I}} J_{\mathcal{I}}(x)$ is positive definite on the null-space of $J_{\mathcal{E}}(x)$, the primal-dual Newton step (50) for (47) is a descent direction for $\theta$. If not, negative-curvature descent directions are easy to obtain.

For the most part, theoretical analyses of these techniques make relatively strong assumptions - a linear independence qualification condition (LICQ) is often required to establish global convergence, while fast local convergence analyses rely on strict complementarity. Because the objective function and the barrier objective function both decrease monotonically with $\mu$ along the exact central path (Fiacco and McCormick, 1968, Wright, 
1992), path-following algorithms for nonlinear programming have a monotone flavour. This is at variance with other methods discussed earlier.

A most disturbing aspect of linesearch-based interior-point methods which use (47) to compute the search direction $d_{x}$ has recently been discovered (Wächter and Biegler, 2000). The issue is that if there is a mixture of equality and inequality constraints, if the former are approximated by linearizations and if feasibility of the latter are controlled by restricting the step along the search direction, the resulting iteration may converge to a worthless infeasible point. This surprising result has caused a reassessment of linesearch methods, and in some cases consideration of filter methods with appropriate acceptance measures as a replacement (Benson, Vanderbei and Shanno, 2002, Wächter and Biegler, 2004). The natural alternative, though, is to consider trust-region-based methods, which fortunately do not suffer from this convergence failure.

In trust-region interior methods for general nonlinear programming, any of the SQPE approaches discussed in $\S 5.2$ are appropriate, but now extra care needs to be taken to cope with the required feasibility of the inequality constraints in (45) or slacks in (46). In particular, in the latter case, it is important that the slack variables do not approach their bounds either prematurely or too rapidly. The obvious SQPE trust-region subproblem would minimize a quadratic approximation to the Lagrangian of (46) - in which as usual a primal-dual approximation $Z_{\mathcal{I}} S_{\mathcal{I}}^{-1}$ to the Hessian of the barrier terms rather than the primal one $\mu S_{\mathcal{I}}^{-2}$ is used-subject to linearized approximations to the constraints within an appropriately-scaled trust region and perhaps a suitable fraction-to-the-boundary constraint. For example, the step $\left(d_{x}, d_{s}\right)$ may be constrained so that

$$
\left\|\left(d_{x}, S^{-1} d_{s}\right)\right\|_{2} \leq \Delta \text { and } s+d_{s} \geq(1-\tau) s
$$

with $0<\tau \lesssim 1$ (Byrd, Gilbert and Nocedal, 2000a, Byrd, Hribar and Nocedal, 1999), or the fraction-to-the-boundary rule may be imposed after the event (Conn et al., 2000b). In general, it is especially important that the shape of the trust region mirrors that of the illconditioned barrier terms (Conn et al., 2000b). As before, the issue of linearized constraint incompatibility - particularly when there is a trust region - is present, and a compositestep strategy as outlined in $§ 5.3 .1$ is appropriate. As in the linesearch case, the penalty parameter $\nu$ must be adjusted as the algorithm proceeds to try to ensure asymptotic feasibility of the constraints, and rules to achieve this within a trust-region framework are known (Byrd et al., 2000a). It is also possible to use the augmented penalty-barrier merit function (53) within such a framework (Gertz and Gill, 2004).

Although primal-dual multiplier estimates $z_{\mathcal{I}}$ are usually preferred to primal ones $\mu S_{\mathcal{I}}^{-1} e$, it is important for global convergence that the former do not differ arbitrarily from the latter. To ensure this property, and to encourage fast asymptotic convergence, generated primal-dual estimates are typically projected into a box containing the primal values. This in turn guarantees proximity of the primal-dual Hessian to the pure primal Hessian, which is also required for fast convergence. An alternative is always to compute least-squares multipliers from an estimate of the optimal active set (Dussault, 1995). 
Some problems may not be defined when the constraints are violated, and methods based on (45) directly respect this requirement. Methods based on (46) may be modified to address it by resetting slacks to ensure that all iterates are strictly feasible. This is sometimes referred to as "feasible mode" (Byrd et al., 2003) and is often used in practice (Byrd et al., 2000a). In a linesearch framework, as soon as an iterate $x_{k}$ strictly satisfies the constraint $c_{i}, i \in \mathcal{I}$, i.e.,

$$
c_{i}\left(x_{k}\right) \geq \epsilon>0,
$$

the $i$-th component of the trial slack variables $s_{i}^{\mathrm{T}}=s_{k i}+d_{s i}$ is reset to $c_{i}\left(x^{\mathrm{T}}\right)=c_{i}\left(x_{k}+d_{x}\right)$. Should the resulting step be rejected by the merit function, a shorter step $\left(d_{x}, d_{s}\right)$ is attempted and the process is repeated. In trust-region frameworks, the situation is more complicated since possible successive increases in the merit function caused by this reset might dominate decreases attempted by the step. It should also be kept in mind that (54) might very well never happen.

In practice it is common to encounter degenerate problems, that is those for which the set of Lagrange multipliers is unbounded or, worse, does not exist - often such problems result from "over-modeling". For instance, it is easily seen that

$$
\underset{x \in \mathbb{R}}{\operatorname{minimize}} f(x) \text { subject to } x^{2}=0
$$

where $f: \mathbb{R} \rightarrow \mathbb{R}$ is such that $f^{\prime}(0) \neq 0$, admits no Lagrange multiplier. To deal with this possibility (1) may be transformed so as to

$$
\begin{aligned}
& \underset{x, s}{\operatorname{minimize}} \phi^{\mathrm{S}}(x, s ; \nu) \stackrel{\text { def }}{=} f(x)+\nu \sum_{i \in \mathcal{E}}\left[c_{i}(x)+2 s_{i}\right]+\nu \sum_{i \in \mathcal{I}} s_{i} \\
& \text { subject to } c_{i}(x)+s_{i} \geq 0 \text { and } s_{i} \geq 0, \text { for all } i \in \mathcal{E} \cup \mathcal{I},
\end{aligned}
$$

in terms of so-called elastic variables $s_{\mathcal{E}} \in \mathbb{R}^{n_{\mathcal{E}}}$ and $s_{\mathcal{I}} \in \mathbb{R}^{n_{\mathcal{I}}}$; the objective $\phi^{\mathrm{S}}(x, s ; \nu)$ is simply a smooth reformulation of the exact $\ell_{1}$-penalty function for (1). This new problem is not only smooth but regular - it satisfies the Mangasarian-Fromovitz constraint qualification, and thus has bounded multipliers, for all fixed $\nu>0$. Furthermore, the problem only involves inequality constraints and is thus well suited to an interior-point approach (Gould, Orban and Toint, 2003b; see also Tits, Wächter, Bakhtiari, Urban and Lawrence, 2003 for a simplified variant).

One other possibility is to balance satisfaction of centrality and feasibility against optimality using a filter. The central idea is to compute a primal-dual step for (49) in a manner similar to that described in (50). But now instead of defining a new iterate by a linesearch along the step, or by some classical trust-region scheme, a two-dimensional filter with conflicting objectives (see $\S 2.1 .3$ )

$$
\begin{aligned}
\theta_{1}\left(x, s_{\mathcal{I}}, y, z_{\mathcal{I}}\right) & =\left\|c_{\mathcal{E}}(x)\right\|+\left\|c_{\mathcal{I}}(x)-s_{\mathcal{I}}\right\|+\left\|Z_{\mathcal{I}} s_{\mathcal{I}}-\frac{z_{\mathcal{I}}^{T} s_{\mathcal{I}}}{n_{\mathcal{I}}} e\right\| \\
\text { and } \quad \theta_{2}\left(x, s_{\mathcal{I}}, y, z_{\mathcal{I}}\right) & =\frac{z_{\mathcal{I}}^{T} s_{\mathcal{I}}}{n_{\mathcal{I}}}+\left\|\nabla_{\left(x, s_{\mathcal{I}}\right)} \ell\left(x, s_{\mathcal{I}}, y, z_{\mathcal{I}}\right)\right\|
\end{aligned}
$$


is used to accept or reject the step; here $\ell\left(x, s_{\mathcal{I}}, y, z_{\mathcal{I}}\right)$ is the Lagrangian of (49). The first objective, $\theta_{1}$, measures feasibility and centrality of the vector $\left(x, s_{\mathcal{I}}, y, z_{\mathcal{I}}\right)$ while $\theta_{2}$ attempts to measure optimality. The resulting algorithm, which decomposes the primal-dual step into normal (towards the central path) and tangential (to the central path) components whose sizes are controlled by a trust-region scheme, is globally convergent to first-order critical points (Ulbrich, Ulbrich and Vicente, 2004).

\subsubsection{Practicalities}

Many practical issues are to be considered with extreme care when implementing pathfollowing methods. Among those, we have already touched on the treatment of indefiniteness, degeneracy, unboundedness, poor scaling and handling of feasible sets with no strict interior. We now briefly comment on two other outstanding issues, the choice of the initial barrier parameter and its update.

The initial value of $\mu$, although irrelevant in theory, is crucial in practice and may determine the success of a method within the allowed limits. Most algorithms set the initial barrier parameter to some prescribed constant value which seems to perform well on average over a large class of problems, e.g., $\mu_{0}=0.1$.

For the formulation (46), if initial values for $s_{\mathcal{I}}$ and $z_{\mathcal{I}}$ are determined using (33), the initial value $\mu_{0}=s^{T} z_{\mathcal{I}} / n_{\mathcal{I}}$ is reminiscent of linear programming (Wright, 1997) to obtain good centrality at the initial point. To take scaling into account and in an attempt to locate nearby points on the central path, one might set $\mu_{0}=\max _{i}\left\|\nabla c_{i}\left(x_{0}\right)\right\|_{\infty}$ and perform a heuristic test by selecting the value of $\mu$ producing the smallest residual in the primaldual system among the values $0.01 \mu 0,0.1 \mu_{0}, \mu_{0}, 10 \mu_{0}$ and $100 \mu_{0}$-this rule is used by interior-point codes in the GALAHAD library (Gould et al., 2003a). Perhaps more usefully, if $P_{J_{\mathcal{E}}(x)}(v)$ denotes the orthogonal projection of $v$ onto the nullspace of $J_{\mathcal{E}}(x)$ and $\phi(x ; \mu)$ is the objective of (45) Gay et al. (1998) suggest computing

$$
\mu_{\mathrm{LS}}=\operatorname{argmin}_{\mu>0}\left\|P_{J_{\mathcal{E}}\left(x_{0}\right)}\left(\nabla_{x} \phi(x ; \mu)\right)\right\|,
$$

and subsequently setting the initial barrier parameter for (45) to the value

$$
\mu_{0}=\min \left(100, \max \left(1,\left|\mu_{\mathrm{LS}}\right|\right)\right) ;
$$

For (46), the same recipe involving $P_{J(x)}$ and the objective $\phi(x, s ; \mu)$ is appropriate.

In short-step, long-step or predictor-corrector methods for linear programming (Wright, 1997) and convex quadratic programming, the barrier parameter is updated using a rule similar to $\mu_{k+1}=\sigma_{k} x_{k}^{T} s_{k} / n_{\mathcal{I}}$, where $0<\sigma_{k}<1$ is a centering parameter. More traditional rules, such as $\mu_{k+1}=\sigma_{k} \mu_{k}$ with $0<\sigma_{k}<1$ are commonplace in nonlinear programming, given that there is no concept of duality gap, and virtually all convergence theory has been established for such rules.

In the framework (45), Gay et al. (1998) suggest the rule

$$
\mu_{k+1}=\min \left(\mu_{k}, \sigma_{k} \frac{c\left(x_{k}\right)^{T} z_{k}}{n_{\mathcal{I}}}\right) \quad \text { where } \quad \sigma_{k}=\min \left(0.2,100 \frac{c\left(x_{k}\right)^{T} z_{k}}{n_{\mathcal{I}}}\right),
$$


where $z_{k}$ are the estimates of the Lagrange multipliers associated to the inequality constraints of (1) at $x_{k}$. This rule is clearly reminiscent of linear programming and enforces that $\left\{\mu_{k}\right\}$ be decreasing. For some problems, this monotone behaviour causes difficulties and, sometimes, failure in practice, and more dynamic rules are investigated, such as the laxer

$$
\mu_{k+1}=\sigma \frac{c\left(x_{k}\right)^{T} z_{k}}{n_{\mathcal{I}}}
$$

with $0<\sigma<1$, which allows the barrier parameter to increase (Bakry, Tapia, Tsuchiya and Zhang, 1996). Similar rules have been used in the framework of (46), using $s_{\mathcal{I} k}^{T} z_{\mathcal{I} k} / n_{\mathcal{I}}$ instead of $c\left(x_{k}\right)^{T} z_{k} / n_{\mathcal{I}}$.

Vanderbei and Shanno (1999) note that in practice, it is important to keep individual complementarity pairs clustered together. Using the formulation (46), they define

$$
\xi_{k}=\frac{\min _{i} s_{k i} z_{k i}}{s_{\mathcal{I} k}^{T} z_{\mathcal{I} k} / n_{\mathcal{I}}}
$$

to measure deviation from complementarity and use the heuristic update

$$
\mu_{k+1}=0.1 \min \left(0.05 \frac{1-\xi_{k}}{\xi_{k}}, 2\right)^{3} \frac{s_{\mathcal{I} k}^{T} z_{\mathcal{I} k}}{n_{\mathcal{I}}}
$$

Such rules have had some success in practice but are unfortunately not covered by convergence theory and can indeed cause failure if $\mu$ becomes too small prematurely or diverges.

\subsubsection{Problems with equilibrium constraints}

Several formulations of mathematical programs with equilibrium constraints (MPECs) are given in the literature. Generalizing mathematical programs with complementarity constraints (MPCCs), their trait is the presence of a constraint of the form

$$
0 \leq F_{1}(x) \perp F_{2}(x) \geq 0
$$

where $F_{1}, F_{2}: \mathbb{R}^{n} \rightarrow \mathbb{R}^{n_{\mathrm{CC}}}$ and, for $x, y \in \mathbb{R}^{n_{\mathrm{CC}}}$, the notation $x \perp y$ is understood componentwise as meaning $x_{i} y_{i}=0$ for all $i=1, \ldots, n_{\mathrm{CC}}$. Such a constraint might originate, e.g. from variational inequalities, optimality conditions of the inner problem in a bilevel setting, or from an economic equilibrium requiring that either the price or the excess production for a product be zero. In game theory, $F_{1}$ and $F_{2}$ might represent the strategy of the leader and the follower, respectively. In design problems, $F_{1}$ is the design while $F_{2}$ is the response of the system. It is easily seen that problems with a constraint of the form (57) violate the Mangasarian-Fromovitz constraint qualification at every feasible point. Such problems thus always have unbounded sets of multipliers which typically consist in rays. We refer the reader to the recent overview (Leyffer, 2003) for references.

Practical implementations able to reliably treat such problems remain rare and in an active development stage and there is much room left for improvement and the advent of 
new methods. Of particular importance is the impact of the formulation of the complementarity constraints on the performance of algorithms. An additional difficulty appears when studying interior methods for (1) with constraints of the form (57) as no central path exists. To circumvent this issue, most practical methods consider a sequence of relaxed problems with nonempty strict interior (Scheel and Scholtes, 2000), and an interior method is applied to them. A rather simple modification of the step described in the filter linesearch interior algorithm of Wächter and Biegler (2004) is described by Raghunathan and Biegler (2003) who perform a single interior-point iteration per relaxed problem. This modification ensures nonsingularity of the step-defining augmented matrix and alleviates the need for centrality conditions. Numerical difficulties may appear for in the limit, the strict interior of the feasible set vanishes. DeMiguel, Friedlander, Nogales and Scholtes (2004) propose an alternative where this limit is nonempty, removing the need to modify the search directions.

Anitescu (2000) reformulates MPCCs with nonempty Lagrange multiplier sets by smoothing an $\ell_{\infty}$-penalty function. The resulting nonlinear program depends on an elastic variable and has an isolated local minimizer at a solution of the MPCC which, under a quadratic growth condition, can be approached with a finite penalty parameter. This last problem may be solved, e.g., using an SQP approach.

Luo, Pang and Ralph (1998) propose a disjunctive approach, in which the feasible region is decomposed in branches, also called local pieces. A single SQP step is performed on the nonlinear program defined by the current piece, and all pieces must be examined. Superlinear convergence holds under uniqueness of the multipliers.

Using elastic variables in a manner similar to Anitescu (2000), Benson, Sen, Shanno and Vanderbei (2003) reformulate the MPCC by smoothing an $\ell_{\infty}$-penalty function. Under strict complementarity, multipliers at a solution are bounded. The algorithm of Vanderbei and Shanno (1999) implemented in the LOQO package is used to solve the penalty subproblems, using an ad-hoc rule to update the penalty parameter.

Convergence properties of algorithms for (57) typically rely on MPCC-specific regularity conditions such as strong stationarity, the so-called MPCC-LICQ, a strong constraint qualification, and the MPCC-SOSC, a specialized second-order condition. A form of strict complementarity usually ensures fast local convergence. For complete details regarding MPCCs and MPECs, we refer the reader to Luo, Pang and Ralph (1996).

Finally, filter methods can also be adapted for the solution of mixed complementarity problems. Ulbrich (2004a) uses a reformulation of the problem into semi-smooth equations, to which a filter method for least-squares (in a variant very close to that described in $\S 2.2$ ) is then applied. Although preliminary experiments are interesting, extensive numerical evidence is still missing and the effectiveness of the approach remains to be confirmed.

\subsubsection{General convex programs}

We finally consider the special case of problems of the form (1), in which $f$ is convex and the constraints define a convex feasible set. Interior methods for such problems inherit 
many properties of those for linear and convex quadratic programming. Algorithms for the latter may therefore relatively painlessly be extended to the former. In particular, the multiple target tracking strategy of Gondzio (1996) generalizes, a key being the reduction of the Newton matrix for the primal-dual equation to a quasi-definite matrix. The method has the particularity of defining one barrier parameter per constraint.

The fact that there is a great deal of well-understood theory covering the convex case, and that efficient algorithms from linear programming carry over does not imply by any means that tracking the central path is an easy task. Indeed, even for infinitely differentiable convex data, the central path can exhibit an infinite number of segments of constant length and assume the shape of an "antenna" or zigzag infinitely (Gilbert, Gonzaga and Karas, 2002),

To control the stepsize, linesearch-based methods for general convex programming use the $\ell_{2}$ merit function (52). The rationale for this approach is that for sufficiently large values of $\nu>0$, the direction $d$ computed from (50) is a descent direction for (52) whenever the problem is strictly convex. The additional difficulty introduced by the use of such a merit function is the need to manage the penalty parameter. For most practical purposes, simple updating rules such as $\nu_{k+1}=10 \nu_{k}$ suffice. More clever rules (approximately) compute the smallest value $\nu_{\min }$ of $\nu$ which makes $d$ a descent direction for the merit function, and set $\nu_{k+1}=10 \nu_{\min }$. The linesearch procedure next determines an appropriate stepsize based on a fraction-to-the-boundary rule and an Armijo-type acceptance condition.

\subsubsection{Software}

Perhaps the most widely-known SQP method is SNOPT (Gill et al., 2002), a worthy successor to the augmented-Lagrangian based MINOS (Murtagh and Saunders, 1982). Both methods are especially designed for the case where there are relatively few degrees of freedom - and most successful in this case - and neither requires second derivatives. The augmented-Lagrangian-based LANCELOT (Conn et al., 1992) operates at the other extreme, being most effective when there are relatively few general constraints, and is capable of running without gradients if necessary-(group) partial separability (Griewank and Toint, 1982b, Conn et al., 1990) allows for the efficient estimation of derivatives. More modern SQP interior-point hybrids like LOQO (Vanderbei and Shanno, 1999), KNITRO (Byrd et al., 2000a) and NLPSPR (Betts and Frank, 1994) are effective regardless of the relative number of (active) constraints. Of the filter-based methods both the trust-region SQP-based FilterSQP (Fletcher and Leyffer, 1998) and the linesearch interior-point-based IPOPT (Wächter and Biegler, 2004) have proved to be robust and efficient. The primaldual method of Forsgren and Gill (1998) is being implemented in the object-oriented code IOTR which acts as a template for implementing interior-point algorithms. Some codesfor example, the augmented-Lagrangian-based PENNON (Kočvara and Stingl, 2003) - have even wider scope, permitting semi-definite matrix constraints. Others, such as CONOPT (Drud, 1994) and LSGRG2 (Smith and Lasdon, 1992), use (generalized) reduced gradient methods not even covered in this survey. A welcome development has certainly been 
the flurry of papers - see for example those just cited - comparing and contrasting rival nonlinear programming packages. At this stage, algorithm development is still so rapid that it is impossible to identify the best method(s). We urge potential users to try the award-winning NEOS server (Dolan, Fourer, Moré and Munson, 2002, Czyzyk et al., 1998)

http://www-neos.mcs.anl.gov

to compare many of the leading contenders.

Turning to convex programming, both MOSEK (Andersen and Andersen, 2000, Andersen and Ye, 1998) - which is based on a homogeneous model (Andersen and Ye, 1999) — and NLPHOPDM (Epelly, Gondzio and Vial, 2000) —which applies a multiple target tracking strategy (Gondzio, 1996) - are designed for general problems, having evolved from linear programming beginnings. The same is true of PDCO (Saunders and Tomlin, 1996), which implements the regularization scheme of $\S 4.3$. PDCO has been successfully used to solve large-scale entropy maximization problems using Shannon's entropy function $S(x)=-\sum_{i} x_{i} \log \left(x_{j}\right)$ as objective and has proved able of solving a maximum entropy model of web traffic with 662, 463 variables and 51, 152 sparse constraints in 12 iterations.

\section{Conclusion}

We have reviewed recent developments in algorithms for large-scale optimization, successively considering the unconstrained, bound-constrained, linearly-constrained and nonlinearly constrained cases. Emphasis has been put on the underlying principles and theoretical underpinnings of the described methods as well as on practical issues and software.

We are aware that, despite our best efforts, the picture remains incomplete and biased by our experience. This is reflected, for instance, in our lack of cover of neighbouring subjects like variational inequalities and nonsmooth problems, despite their intrinsic interest. It is nevertheless hoped that the overview presented will make the field of nonlinear programming and its application to solving large problems easier to understand, both for scholars and practitioners.

\section{Acknowledgments}

The work of the first author was supported by the EPSRC grant GR/S42170, and that of the second author by NSERC grant RGPIN299010-04 and PIED grant 131FR88. The work of the third author has been conducted in the framework of the Interuniversity Attraction Poles Programme of the Belgian Science Policy Agency. The authors are indebted to Annick Sartenaer for her comments on a draft of the manuscript. 


\section{References}

M. Al-Baali (2003), Quasi-Newton algorithms for large-scale nonlinear least-squares, in Di Pillo and Murli (2003), pp. 1-21.

E. D. Andersen and K. D. Andersen (2000), The MOSEK interior point optimizer for linear programming: an implementation of the homogeneous algorithm, in High Performance Optimization (T. T. H. Frenk, K. Roos and S. Zhang, eds), Kluwer Academic Publishers, pp. 197-232.

E. D. Andersen and Y. Ye (1998), 'A computational study of the homogeneous algorithm for large-scale convex optimization', Computational Optimization and Application 10, 243-269.

E. D. Andersen and Y. Ye (1999), 'On a homogeneous algorithm for the monotone complementarity problem', Mathematical Programming 84(2), 375-399.

M. Anitescu (2000), On using the elastic mode in nonlinear programming approaches to mathematical programs with complementarity constraints, Preprint ANL/MCSP864-1200, Argonne National Laboratory, Illinois, USA.

E. Arian, M. Fahl and E. W. Sachs (2000), Trust-region proper orthogonal decomposition for flow control, Technical Report 2000-25, Institute for Computer Applications in Science and Engineering, NASA Langley Research Center Hampton, Virginia, USA.

A. S. El Bakry, R. A. Tapia, T. Tsuchiya and Y. Zhang (1996), 'On the formulation and theory of newton interior point methods for nonlinear programming', Journal of Optimization Theory and Applications 89(3), 507-541.

R. E. Bank, P. E. Gill and R. F. Marcia (2003), Interior point methods for a class of elliptic variational inequalities, in Biegler, Ghattas, Heinkenschloss and Van Bloemen Waanders (2003), pp. 218-235.

H. Y. Benson, A. Sen, D. F. Shanno and R. J. Vanderbei (2003), Interior-point algorithms, penalty methods and equilibrium problems, Technical Report ORFE-03-02, Operations Research and Financial Engineering, Princeton University.

H. Y. Benson, R. J. Vanderbei and D. F. Shanno (2002), 'Interior-Point Methods for Nonconvex Nonlinear Programming: Filter Methods and Merit Functions', Computational Optimization and Applications 23, 257-272.

S. J. Benson, L. C. McInnes, J. Moré and J. Sarich (2004), Scalable algorithms in optimization: Computational experiments, Preprint ANL/MCS-P1175-0604, Mathematics and Computer Science, Argonne National Laboratory, Argonne, Illinois, USA. To appear in the Proceedings of the 10th AIAA/ISSMO Multidisciplinary Analysis and Optimization (MA\&O) Conference, August 30 - September 1, 2004. 
D. P. Bertsekas (1976), 'On the Goldstein-Levitin-Poljak gradient projection method', IEEE Transactions on Automatic Control AC-21, 174-184.

D. P. Bertsekas (1995), Nonlinear Programming, Athena Scientific, Belmont, Massachussetts, USA.

J. T. Betts and S. O. Erb (2003), 'Optimal low thurst trajectory to the moon', SIAM Journal on Applied Dynamical Systems 2(2), 144-170.

J. T. Betts and P. D. Frank (1994), 'A sparse nonlinear optimization algorithm', Journal of Optimization Theory and Applications 82(3), 519-541.

T. Biegler, O. Ghattas, M. Heinkenschloss and B. Van Bloemen Waanders, eds (2003), High performance algorithms and software for nonlinear optimization, Springer Verlag, Heidelberg, Berlin, New York.

G. Biros and O. Ghattas (2000), 'A Lagrange-Newton-Krylov-Schur method for PDEconstrained optimization', SIAG/Optimiztion Views-and-News 11(2), 12-18.

R. E. Bixby, M. Fenlon, Z. Gu, E. Rothberg and R. Wunderling (2000), MIP: theory and practice - closing the gap, in System Modelling and Optimization, Methods, Theory and Applications (M. J. D. Powell and S. Scholtes, eds), Kluwer Academic Publishers, Dordrecht, The Netherlands, pp. 10-49.

P. T. Boggs and J. W. Tolle (1995), 'Sequential quadratic programming', Acta Numerica $4,1-51$.

P. T. Boggs and J. W. Tolle (2000), 'Sequential quadratic programming for large-scale nonlinear optimization', Computational and Applied Mathematics 124, 123-137.

P. T. Boggs, P. D. Domich, J. E. Rogers and C. Witzgall (1996), 'An interior point method for general large scale quadratic programming problems', Annals of Operations Research 62, 419-437.

P. T. Boggs, A. J. Kearsley and J. W. Tolle (1999a), 'A global convergence analysis of an algorithm for large scale nonlinear programming problems', SIAM Journal on Optimization 9(4), 833-862.

P. T. Boggs, A. J. Kearsley and J. W. Tolle (1999b), 'A practical algorithm for general large scale nonlinear optimization problems', SIAM Journal on Optimization 9(3), $755-778$.

J. F. Bonnans, J.-Ch. Gilbert, C. Lemaréchal and C. Sagastizábal (1997), Optimisation Numérique - Aspects théoriques et pratiques, Mathématiques \& Applications 27, Springer. 
J. M. Borwein (1982), 'Necessary and sufficient conditions for quadratic minimality', Numerical Functional Analysis and Optimization 5, 127-140.

J. H. Bramble (1993), Multigrid Methods, Longman Scientific and Technical, New York.

M. A. Branch, T. F. Coleman and Y. Li (1999), 'A subspace, interior and conjugate gradient method for large-scale bound-constrained minimization problems', SIAM Journal on Scientific Computing 21(1), 1-23.

A. Brandt (1977), 'Multi-level adaptative solutions to boundary value problems', Mathematics of Computation 31(138), 333-390.

W. L. Briggs, V. E. Henson and S. F. McCormick (2000), A Multigrid Tutorial, 2nd edn, SIAM, Philadelphia, USA.

A. Brooke, D. Kendrick and A. Meeraus (1988), GAMS: a User's Guide, The Scientific Press, Redwood City, USA.

J. V. Burke and J. J. Moré (1988), 'On the identification of active constraints', SIAM Journal on Numerical Analysis 25(5), 1197-1211.

J. V. Burke and J. J. Moré (1994), 'Exposing constraints', SIAM Journal on Optimization 4(3), 573-595.

J. V. Burke and A. Weigmann (1997), Notes on limited memory BFGS updating in a trust-region framework, Technical report, Department of Mathematics, University of Washington, Seattle, Washington, USA.

J. V. Burke, J. J. Moré and G. Toraldo (1990), 'Convergence properties of trust region methods for linear and convex constraints', Mathematical Programming 47(3), 305336.

R. H. Byrd, J.-Ch. Gilbert and J. Nocedal (2000a), 'A trust region method based on interior point techniques for nonlinear programming', Mathematical Programming Series A 89, 149-185.

R. H. Byrd, J.-Ch. Gilbert and J. Nocedal (2000b), 'A trust region method based on interior point techniques for nonlinear programming', Mathematical Programming 89(1), 149-185.

R. H. Byrd, N. I. M. Gould, J. Nocedal and R. A. Waltz (2004a), 'An algorithm for nonlinear optimization using linear programming and equality constrained subproblems', Mathematical Programming, Series B 100(1), 27-48.

R. H. Byrd, N. I. M. Gould, J. Nocedal and R. A. Waltz (2004b), On the convergence of successive linear-quadratic programming algorithms, Technical Report RAL-TR2004-032, Rutherford Appleton Laboratory, Chilton, Oxfordshire, England. 
R. H. Byrd, M. E. Hribar and J. Nocedal (1999), 'An interior point method for large scale nonlinear programming', SIAM Journal on Optimization 9(4), 877-900.

R. H. Byrd, P. Lu, J. Nocedal and C. Zhu (1995), 'A limited memory algorithm for bound constrained optimization', SIAM Journal on Scientific Computing 16(5), 1190-1208.

R. H. Byrd, J. Nocedal and R. B. Schnabel (1994), 'Representations of quasi-Newton matrices and their use in limited memory methods', Mathematical Programming 63(2), 129-156.

R. H. Byrd, J. Nocedal and R. A. Waltz (2003), 'Feasible interior methods using slacks for nonlinear optimization', Computational Optimization and Applications 26, 35-61.

P. H. Calamai and J. J. Moré (1987), 'Projected gradient methods for linearly constrained problems', Mathematical Programming 39(1), 93-116.

Y. Chabrillac and J.-P. Crouzeix (1984), 'Definiteness and semidefiniteness of quadratic forms revisited', Linear Algebra and its Applications 63, 283-292.

C. M. Chin and R. Fletcher (2003), 'On the global convergence of an SLP-filter algorithm that takes EQP steps', Mathematical Programming 96(1), 161-177.

T. F. Coleman (1994), Linearly constrained optimization and projected preconditioned conjugate gradients, in Proceedings of the Fifth SIAM Conference on Applied Linear Algebra (J. Lewis, ed.), SIAM, Philadelphia, USA, pp. 118-122.

T. F. Coleman and A. R. Conn (1982), 'Nonlinear programming via an exact penalty function method : Asymptotic analysis', Mathematical Programming 24(3), 123-136.

T. F. Coleman and L. A. Hulbert (1989), 'A direct active set algorithm for large sparse quadratic programs with simple bounds', Mathematical Programming, Series B 45(3), 373-406.

T. F. Coleman and Y. Li, eds (1990), Large Scale Numerical Optimization, SIAM, Philadelphia, USA.

T. F. Coleman and Y. Li (1994), 'On the convergence of interior-reflective Newton methods for nonlinear minimization subject to bounds', Mathematical Programming 67(2), 189-224.

T. F. Coleman and Y. Li (1996), 'An interior trust region approach for nonlinear minimization subject to bounds', SIAM Journal on Optimization 6(2), 418-445.

T. F. Coleman and Y. Li (2000), 'A trust region and affine scaling interior point method for nonconvex minimization with linear inequality constraints', Mathematical Programming, Series A 88, 1-31. 
B. Colson and Ph. L. Toint (2003), Optimizing partially separable functions without derivatives, Technical Report 03/20, Department of Mathematics, University of Namur, Namur, Belgium.

A. R. Conn, N. I. M. Gould and Ph. L. Toint (1988a), 'Global convergence of a class of trust region algorithms for optimization with simple bounds', SIAM Journal on Numerical Analysis 25(2), 433-460. See also same journal 26, 764-767, 1989.

A. R. Conn, N. I. M. Gould and Ph. L. Toint (1988b), 'Testing a class of methods for solving minimization problems with simple bounds on the variables', Mathematics of Computation 50, 399-430.

A. R. Conn, N. I. M. Gould and Ph. L. Toint (1990), An introduction to the structure of large scale nonlinear optimization problems and the LANCELOT project, in Computing Methods in Applied Sciences and Engineering (R. Glowinski and A. Lichnewsky, eds), SIAM, Philadelphia, USA, pp. 42-51.

A. R. Conn, N. I. M. Gould and Ph. L. Toint (1992), LANCELOT: a Fortran package for Large-scale Nonlinear Optimization (Release A), Springer Series in Computational Mathematics, Springer Verlag, Heidelberg, Berlin, New York.

A. R. Conn, N. I. M. Gould and Ph. L. Toint (1994), Large-scale nonlinear constrained optimization: a current survey, in Algorithms for Continuous Optimization: The State of the Art (E. Spedicato, ed.), Vol. 434 of NATO ASI Series C: Mathematical and Physical Sciences, Kluwer Academic Publishers, Dordrecht, The Netherlands, pp. 287-332.

A. R. Conn, N. I. M. Gould and Ph. L. Toint (1996), 'Numerical experiments with the LANCELOT package (Release A) for large-scale nonlinear optimization', Mathematical Programming, Series A 73(1), 73-110.

A. R. Conn, N. I. M. Gould and Ph. L. Toint (1997), Methods for nonlinear constraints in optimization calculations, in Duff and Watson (1997), pp. 363-390.

A. R. Conn, N. I. M. Gould and Ph. L. Toint (2000a), Trust-Region Methods, SIAM, Philadelphia, USA.

A. R. Conn, N. I. M. Gould, D. Orban and Ph. L. Toint (2000b), 'A primal-dual trustregion algorithm for non-convex nonlinear programming', Mathematical Programming B 87(2), 215-249.

B. L. Contesse (1980), 'Une caractérisation complète des minima locaux en programmation quadratique', Numerische Mathematik 34(3), 315-332.

CPLEX 6.0 (1998), High-performance linear, integer and quadratic programming software, ILOG SA, Gentilly, France. www.cplex.com. 
J. Czyzyk, M. Mesnier and J. Moré (1998), 'The NEOS server', IEEE Journal on Computational Science and Engineering 5, 68-75.

Y. H. Dai and Y. Yuan (2000), 'A nonlinear conjugate gradient method with a strong global convergence property', SIAM Journal on Optimization 10(1), 177-182.

R. S. Dembo and T. Steihaug (1983), 'Truncated-Newton algorithms for large-scale unconstrained optimization', Mathematical Programming 26(2), 190-212.

R. S. Dembo, S. C. Eisenstat and T. Steihaug (1982), 'Inexact-Newton methods', SIAM Journal on Numerical Analysis 19(2), 400-408.

A.-V. DeMiguel, M. P. Friedlander, F. J. Nogales and S. Scholtes (2004), An interiorpoint method for MPECs based on strictly feasible relaxations, Technical Report ANL/MCS-P1150-0404, Argonne National Laboratory, Illinois, USA.

N. Deng, Y. Xiao and F. Zhou (1993), 'Nonmonotonic trust region algorithms', Journal of Optimization Theory and Applications 76(2), 259-285.

J. E. Dennis and R. B. Schnabel (1983), Numerical methods for unconstrained optimization and nonlinear equations, Prentice-Hall, Englewood Cliffs, USA. Reprinted as Classics in Applied Mathematics 16, SIAM, Philadelphia, USA.

J. E. Dennis, D. M. Gay and R. E. Welsh (1981), 'An adaptive nonlinear least squares algorithm', ACM Transactions on Mathematical Software 7(3), 348-368.

G. Di Pillo and F. Gianessi, eds (1996), Nonlinear Optimization and Applications, Plenum Publishing, New York.

G. Di Pillo and F. Gianessi, eds (1999), Nonlinear Optimization and Applications 2, Kluwer Academic Publishers, Dordrecht, The Netherlands.

G. Di Pillo and A. Murli, eds (2003), High Performance Algorithms and Software in Nonlinear Optimization, Kluwer Academic Publishers, Dordrecht, The Netherlands.

E. Dolan (2001), The NEOS server 4.0 administrative guide, Technical Memorandum ANL/MCS-TM-250, The Mathematical and Computer Science Division, Argonne National Laboratory, Argonne, IL.

E. D. Dolan, R. Fourer, J. J. Moré and T. S. Munson (2002), 'Computing a trust region step', SIAM News 35(5), 8-9.

A. S. Drud (1994), 'CONOPT-a large scale GRG code', ORSA Journal on Computing 6, 207-216.

I. Duff and A. Watson, eds (1997), The State of the Art in Numerical Analysis, Oxford University Press, Oxford, England. 
J. C. Dunn (1981), 'Global and asymptotic convergence rate estimates for a class of projected gradient processes', SIAM Journal on Control and Optimization 19, 368400.

J.-P. Dussault (1995), 'Numerical stability and efficiency of penalty algorithms', SIAM Journal on Numerical Analysis 32(1), 296-317.

O. Epelly, J. Gondzio and J.-P. Vial (2000), An interior-point solver for smooth convex optimization with an application to environmental-energy-economic models, Technical Report 2000.08, Logilab, HEC, University of Geneva, Switzerland.

F. Facchinei, J. Judice and J. Soares (1998), 'An active set newton algorithm for large-scale nonlinear programs with box constraints', SIAM Journal on Optimization 8(1), 158-186.

F. Facchinei, S. Lucidi and L. Palagi (2002), 'A truncated Newton algorithm for large scale box constrained optimization', SIAM Journal on Optimization 12(4), 1100-1125.

M. Fahl and E. Sachs (2003), Reduced order modelling approaches to PDE-constrained optimization based on proper orthogonal decomposition, in Biegler et al. (2003), pp. 268-281.

M. C. Ferris and T. S. Munson (2000), Interior-point methods for massive support vector machines, Data Mining Institute Technical Report 00-05, Computer Science Department, University of Wisconsin, Madison, WI, USA.

A. V. Fiacco and G. P. McCormick (1968), Nonlinear Programming: Sequential Unconstrained Minimization Techniques, J. Wiley and Sons, Chichester, England. Reprinted as Classics in Applied Mathematics, SIAM, Philadelphia, USA, 1990.

M. Fisher (1998), Minimization algorithms for variational data assimilation, in Recent Developments in Numerical Methods for Atmospheric Modelling, ECMWF, pp. 364385.

R. Fletcher (1971), 'A general quadratic programming algorithm', Journal of the Institute of Mathematics and its Applications 7, 76-91.

R. Fletcher (1981), Practical Methods of Optimization: Constrained Optimization, J. Wiley and Sons, Chichester, England.

R. Fletcher (1987a), Practical Methods of Optimization, second edn, J. Wiley and Sons, Chichester, England.

R. Fletcher (1987b), Recent developments in linear and quadratic programming, in The State of the Art in Numerical Analysis (A. Iserles and M. J. D. Powell, eds), Oxford University Press, Oxford, England, pp. 213-243. 
R. Fletcher (2000), 'Stable reduced hessian updates for indefinite quadratic programming', Mathematical Programming 87(2), 251-264.

R. Fletcher and S. Leyffer (1998), User manual for filterSQP, Numerical Analysis Report NA/181, Department of Mathematics, University of Dundee, Dundee, Scotland.

R. Fletcher and S. Leyffer (2002), 'Nonlinear programming without a penalty function', Mathematical Programming 91(2), 239-269.

R. Fletcher and C. M. Reeves (1964), 'Function minimization by conjugate gradients', Computer Journal 7, 149-154.

R. Fletcher and E. Sainz de la Maza (1989), 'Nonlinear programming and nonsmooth optimization by successive linear programming', Mathematical Programming 43(3), 235256.

R. Fletcher, N. I. M. Gould, S. Leyffer, Ph. L. Toint and A. Wächter (2002a), 'Global convergence of trust-region SQP-filter algorithms for nonlinear programming', SIAM Journal on Optimization 13(3), 635-659.

R. Fletcher, S. Leyffer and Ph. L. Toint (2002b), 'On the global convergence of a filter-SQP algorithm', SIAM Journal on Optimization 13(1), 44-59.

A. Forsgren (2002), 'Inertia-controlling factorizations for optimization algorithms', $A p$ plied Numerical Mathematics 43(1-2), 91-107.

A. Forsgren and P. E. Gill (1998), 'Primal-dual interior methods for nonconvex nonlinear programming', SIAM Journal on Optimization 8(4), 1132-1152.

A. Forsgren and W. Murray (1993), 'Newton methods for large-scale linear equalityconstrained minimization', SIAM Journal on Matrix Analysis and Applications $\mathbf{1 4}(2), 560-587$.

A. Forsgren, P. E. Gill and M. H. Wright (2002), 'Interior-point methods for nonlinear optimization', SIAM Review 44, 525-597.

R. Fourer, D. M. Gay and B. W. Kernighan (2003), AMPL: A modeling language for mathematical programming, second edn, Brooks/Cole-Thompson Learning, Pacific Grove, California, USA.

M. P. Friedlander and M. A. Saunders (2005), 'A globally convergent linearly constrained Lagrangian method for nonlinear optimization', SIAM Journal on Optimization p. to appear.

D. M. Gay, M. L. Overton and M. H. Wright (1998), A primal-dual interior method for nonconvex nonlinear programming, in Advances in Nonlinear Programming (Y. Yuan, ed.), Kluwer Academic Publishers, Dordrecht, The Netherlands, pp. 31-56. 
E. M. Gertz and Ph. E. Gill (2004), 'A primal-dual trust region algorithm for nonlinear optimization', Mathematical Programming, Series A 100(1), 49-94.

E. M. Gertz and S. J. Wright (2003), 'Object-oriented software for quadratic programming', Transactions of the ACM on Mathematical Software 29(1), 58-81.

E. M. Gertz, J. Nocedal and A. Sartenaer (2003), A starting-point strategy for nonlinear interior methods, Technical Report OTC 2003/4, Optimization Technology Center, Evanston, IL, USA.

J.-Ch. Gilbert and C. Lemaréchal (1989), 'Some numerical experiments with variablestorage quasi-Newton algorithms', Mathematical Programming, Series B 45(3), 407435.

J.-Ch. Gilbert and J. Nocedal (1992), 'Global convergence properties of conjugate gradient methods for optimization', SIAM Journal on Optimization 2(1), 21-42.

J.-Ch. Gilbert, C. C. Gonzaga and E. Karas (2002), Examples of ill-behaved central paths in convex optimization, Technical Report 4179, INRIA, Rocquencourt, Le Chesnay, France.

P. E. Gill, W. Murray and M. A. Saunders (2002), 'SNOPT: An SQP algorithm for largescale constrained optimization', SIAM Journal on Optimization 12(4), 979-1006.

P. E. Gill, W. Murray and M. H. Wright (1981), Practical Optimization, Academic Press, London, England.

P. E. Gill, W. Murray, M. A. Saunders and M. H. Wright (1990), A Schur-complement method for sparse quadratic programming, in Reliable Scientific Computation (M. G. Cox and S. J. Hammarling, eds), Oxford University Press, Oxford, England, pp. 113138.

P. E. Gill, W. Murray, M. A. Saunders and M. H. Wright (1991), 'Inertia-controlling methods for general quadratic programming', SIAM Review 33(1), 1-36.

M. J. Goldsmith (1999), Sequential quadratic programming methods based on indefinite Hessian approximations, PhD thesis, Dept of Management Science and Engineering, Stanford University, California, USA.

J. Gondzio (1996), 'Multiple centrality corrections in a primal-dual method for linear programming', Computational Optimization and Applications 6, 137-156.

J. Gondzio (1997), 'Presolve analysis of linear programs prior to applying an interior point method', INFORMS Journal on Computing 9(1), 73-91. 
J. Gondzio and A. Grothey (2003a), Parallel interior point solver for structured quadratic programs: Application to financial planning problems, Technical Report MS-03-001, School of Mathematics, University of Edinburgh, Scotland.

J. Gondzio and A. Grothey (2003b), 'Reoptimization with the primal-dual interior point method', SIAM Journal on Optimization 13(3), 842-864.

C. C. Gonzaga, E. Karas and M. Vanti (2003), 'A globally convergent filter method for nonlinear programming', SIAM Journal on Optimization 14(3), 646-669.

N. I. M. Gould (1985), 'On practical conditions for the existence and uniqueness of solutions to the general equality quadratic-programming problem', Mathematical Programming 32(1), 90-99.

N. I. M. Gould (1986), 'On the accurate determination of search directions for simple differentiable penalty functions', IMA Journal of Numerical Analysis 6, 357-372.

N. I. M. Gould (1991), 'An algorithm for large-scale quadratic programming', IMA Journal of Numerical Analysis 11(3), 299-324.

N. I. M. Gould (1999), 'On modified factorizations for large-scale linearly-constrained optimization', SIAM Journal on Optimization 9(4), 1041-1063.

N. I. M. Gould (2003), 'Some reflections on the current state of active-set and interior point methods for constrained optimization', SIAG/OPT Views-and-News 14(1), 2-7.

N. I. M. Gould, and Ph. L. Toint (2000a), A quadratic programming bibliography, Numerical Analysis Group Internal Report 2000-1, Rutherford Appleton Laboratory, Chilton, Oxfordshire, England.

N. I. M. Gould and Ph. L. Toint (2000), SQP methods for large-scale nonlinear programming, in System Modelling and Optimization, Methods, Theory and Applications (M. J. D. Powell and S. Scholtes, eds), Kluwer Academic Publishers, Dordrecht, The Netherlands, pp. 149-178.

N. I. M. Gould and Ph. L. Toint (2002a), 'An iterative working-set method for large-scale non-convex quadratic programming', Applied Numerical Mathematics 43(1-2), 109128.

N. I. M. Gould and Ph. L. Toint (2002b), Numerical methods for large-scale non-convex quadratic programming, in Trends in Industrial and Applied Mathematics (A. H. Siddiqi and M. Kočvara, eds), Kluwer Academic Publishers, Dordrecht, The Netherlands, pp. 149-179. 
N. I. M. Gould and Ph. L. Toint (2003a), FILTRANE, a Fortran 95 filter-trust-region package for solving systems of nonlinear equalities, nonlinear inequalities and nonlinear least-squares problems, Technical Report 03/15, Rutherford Appleton Laboratory, Chilton, Oxfordshire, England.

N. I. M. Gould and Ph. L. Toint (2003b), Global convergence of a hybrid trust-region SQP-filter algorithm for general nonlinear programming, in System Modeling and Optimization XX (E. Sachs and R. Tichatschke, eds), Kluwer Academic Publishers, Dordrecht, The Netherlands, pp. 23-54.

N. I. M. Gould and Ph. L. Toint (2004a), Global convergence of a non-monotone trust-region filter algorithm for nonlinear programming, in Proceedings of the 2004 Gainesville Conference on Multilevel Optimization (W. Hager, ed.), Kluwer Academic Publishers, Dordrecht, The Netherlands. (To appear).

N. I. M. Gould and Ph. L. Toint (2004b), How mature is nonlinear optimization?, in Applied Mathematics Entering the 21st Century: Invited Talks from the ICIAM 2003 Congress (J. M. Hill and R. Moore, eds), SIAM, Philadelphia, USA. (To appear).

N. I. M. Gould and Ph. L. Toint (2004c), 'Preprocessing for quadratic programming', Mathematical Programming, Series B 100(1), 95-132.

N. I. M. Gould, M. E. Hribar and J. Nocedal (2001), 'On the solution of equality constrained quadratic problems arising in optimization', SIAM Journal on Scientific Computing 23(4), 1375-1394.

N. I. M. Gould, S. Leyffer and Ph. L. Toint (2004a), 'A multidimensional filter algorithm for nonlinear equations and nonlinear least-squares', SIAM Journal on Optimization. (To appear).

N. I. M. Gould, S. Lucidi, M. Roma and Ph. L. Toint (1999), 'Solving the trust-region subproblem using the Lanczos method', SIAM Journal on Optimization 9(2), 504-525.

N. I. M. Gould, S. Lucidi, M. Roma and Ph. L. Toint (2000b), 'Exploiting negative curvature directions in linesearch methods for unconstrained optimization', Optimization Methods and Software 14(1-2), 75-98.

N. I. M. Gould, D. Orban and Ph. L. Toint (2003a), 'GALAHAD - a library of thread-safe Fortran 90 packages for large-scale nonlinear optimization', ACM Transactions on Mathematical Software 29(4), 353-372.

N. I. M. Gould, D. Orban and Ph. L. Toint (2003b), An interior-point $\ell_{1}$-penalty method for nonlinear optimization, Technical Report RAL-TR-2003-0xx, Rutherford Appleton Laboratory, Chilton, Oxfordshire, England. 
N. I. M. Gould, C. Sainvitu and Ph. L. Toint (2004b), A filter-trust-region method for unconstrained optimization, Technical Report 04/03, Department of Mathematics, University of Namur, Namur, Belgium.

S. Gratton, A. Sartenaer and Ph. L. Toint (2004), Recursive trust-region methods for multilevel nonlinear optimization (Part I): Global convergence and complexity, Technical Report 04/06, Department of Mathematics, University of Namur, Namur, Belgium.

A. Griewank (2000), Evaluating derivatives: principles and techniques of algorithmic differentiation, number 19 in 'Frontiers in Applied Mathematics', SIAM, Philadelphia, USA.

A. Griewank and Ph. L. Toint (1982a), 'Local convergence analysis for partitioned quasiNewton updates', Numerische Mathematik 39, 429-448.

A. Griewank and Ph. L. Toint (1982b), On the unconstrained optimization of partially separable functions, in Nonlinear Optimization 1981 (M. J. D. Powell, ed.), Academic Press, London, England, pp. 301-312.

A. Griewank and Ph. L. Toint (1982c), 'Partitioned variable metric updates for large structured optimization problems', Numerische Mathematik 39, 119-137.

R. E. Griffith and R. A. Stewart (1961), 'A nonlinear programming technique for the optimization of continuous processing systems', Management Science 7, 379-392.

L. Grippo, F. Lampariello and S. Lucidi (1986), 'A nonmonotone line search technique for Newton's method', SIAM Journal on Numerical Analysis 23(4), 707-716.

L. Grippo, F. Lampariello and S. Lucidi (1989), 'A truncated Newton method with nonmonotone line search for unconstrained optimization', Journal of Optimization Theory and Applications 60(3), 401-419.

W. Gropp and J. Moré (1997), Optimization environments and the NEOS server, in $A p$ proximation theory and optimization (M. D. Buhmann and A. Iserles, eds), Cambridge University Press, pp. 167-182.

C. Guéret, C. Prins and M. Seveaux (2002), Applications of Optimization with Xpress$M P$, Dash Optimization. www.dashoptimization.com.

M. Gulliksson, I. Söderkvist and P.-A. Wedin (1997), 'Algorithms for constrained and weighted nonlinear least-squares', SIAM Journal on Optimization 7(1), 208-224.

W. Hackbusch (1995), Multi-grid Methods and Applications, number 4 in 'Springer Series in Computational Mathematics', Springer Verlag, Heidelberg, Berlin, New York.

W. W. Hager (2001), 'Minimizing a quadratic over a sphere', SIAM Journal on Optimization 12(1), 188-208. 
W. W. Hager and H. Zhang (2003), CG_DESCENT, a conjugate-gradient method with guaranteed descent, Technical report, Department of Mathematics, University of Florida, Gainesville, USA.

W. W. Hager, D. W. Hearn and P. M. Pardalos, eds (1994), Large Scale Optimization: State of the Art, Kluwer Academic Publishers, Dordrecht, The Netherlands.

M. Heinkenschloss, M. Ulbrich and S. Ulbrich (1999), 'Superlinear and quadratic convergence of affine-scaling interior-point Newton methods for problems with simple bounds without strict complementarity assumption', Mathematical Programming 86(3), 615635.

M. R. Hestenes and E. Stiefel (1952), 'Methods of conjugate gradients for solving linear systems', Journal of Research of the National Bureau of Standards 49, 409-436.

W. Hock and K. Schittkowski (1981), Test Examples for Nonlinear Programming Codes, Springer Verlag, Heidelberg, Berlin, New York. Lectures Notes in Economics and Mathematical Systems 187.

B. Jansen, C. Roos, T. Terlaky and J.-Ph. Vial (1996), 'Primal-dual target following algorithms for linear programming', Annals of Operations Research 62, 197-231.

L. C. Kaufman (1999), 'Reduced storage, quasi-Newton trust region approaches to function optimization', SIAM Journal on Optimization 10(1), 56-69.

M. Kočvara and M. Stingl (2003), 'PENNON, a code for nonconvex nonlinear and semidefinite programming', Optimization Methods and Software 18(3), 317-333.

M. Lalee, J. Nocedal and T. D. Plantenga (1998), 'On the implementation of an algorithm for large-scale equality constrained optimization', SIAM Journal on Optimization 8(3), 682-706.

R. D. Leone, A. Murli, P. M. Pardalos and G. Toraldo, eds (1998), High Performance Algorithms and Software in Nonlinear Optimization, Kluwer Academic Publishers, Dordrecht, The Netherlands.

M. Lescrenier (1991), 'Convergence of trust region algorithms for optimization with bounds when strict complementarity does not hold', SIAM Journal on Numerical Analysis 28(2), 476-495.

E. S. Levitin and B. T. Polyak (1966), 'Constrained minimization problems', U.S.S.R. Computational Mathematics and Mathematical Physics 6, 1-50.

A. S. Lewis and M. L. Overton (1996), 'Eigenvalue optimization', Acta Numerica 5, 149190. 
M. Lewis and S. G. Nash (2002), Practical aspects of multiscale optimization methods for vlsicad, in Multiscale Optimization and VLSI/CAD (J. Cong and J. R. Shinnerl, eds), Kluwer Academic Publishers, Dordrecht, The Netherlands, pp. 265-291.

M. Lewis and S. G. Nash (2004), 'Model problems for the multigrid optimization of systems governed by differential equations', SIAM Journal on Scientific Computing. (To appear).

S. Leyffer (2003), 'Mathematical Programs with Complementarity Constraints', SIAG/OPT Views-and-News 14(1), 15-18.

C. Lin and J. J. Moré (1999a), 'Incomplete Cholesky factorizations with limited memory', SIAM Journal on Scientific Computing 21(1), 24-45.

C. Lin and J. J. Moré (1999b), 'Newton's method for large bound-constrained optimization problems', SIAM Journal on Optimization 9(4), 1100-1127.

D. C. Liu and J. Nocedal (1989), 'On the limited memory BFGS method for large-scale optimization', Mathematical Programming, Series B 45(3), 503-528.

D. G. Luenberger (1984), Linear and nonlinear programming, second edn, Addison-Wesley Publishing Company, Reading, Massachusetts, USA.

L. Lukšan (1993), 'Inexact trust region method for large sparse nonlinear least-squares', Kybernetica 29(4), 305-324.

L. Lukšan (1994), 'Inexact trust region method for large sparse systems of nonlinear equations', Journal of Optimization Theory and Applications 81(3), 569-590.

L. Lukšan (1996), 'Hybrid methods for large sparse nonlinear least-squares', Journal of Optimization Theory and Applications 89(3), 575-595.

L. Lukšan and J. Vlček (1998), 'Indefinitely preconditioned inexact Newton method for large sparse equality constrained nonlinear programming problems', Numerical Linear Algebra with Applications 5(3), 219-247.

Z.-Q. Luo, J. S. Pang and D. Ralph (1996), Mathematical Programs with Equilibrium Constraints, Cambridge Univeristy Press, Cambridge, UK.

Z.-Q. Luo, J. S. Pang and D. Ralph (1998), Piecewise sequential quadratic programming for mathematical programs with complementarity constraints, in Multilevel Optimization: Complexity and Applications (A. M. et al., ed.), Kluwer Academic Publishers.

O. L. Mangasarian (1980), 'Locally unique solutions of quadratic programs, linear and non-linear complementarity problems', Mathematical Programming, Series B 19(2), 200-212. 
N. Maratos (1978), Exact penalty function algorithms for finite-dimensional and control optimization problems, PhD thesis, University of London, London, England.

M. Marazzi and J. Nocedal (2001), Feasibility control in nonlinear optimization, in Foundations of Computational Mathematics (A. DeVore, A. Iserles and E. Suli, eds), London Mathematical Society Lecture Note Series 284, Cambridge University Press, Cambridge, England, pp. 125-154.

D. Q. Mayne and E. Polak (1976), 'Feasible directions algorithms for optimisation problems with equality and inequality constraints', Mathematical Programming 11(1), 6780 .

S. Mehrotra (1992), 'On the implementation of a primal-dual interior point method', SIAM Journal on Optimization 2, 575-601.

J. L. Morales and J. Nocedal (2000), 'Automatic preconditioning by limited memory quasi-newton updating', SIAM Journal on Optimization 10(4), 1079-1096.

J. J. Moré (2003), 'Terascale optimal PDE solvers', Talk at the ICIAM 2003 Conference in Sydney.

J. J. Moré and D. C. Sorensen (1983), 'Computing a trust region step', SIAM Journal on Scientific and Statistical Computing 4(3), 553-572.

J. J. Moré and D. J. Thuente (1994), 'Line search algorithms with guaranteed sufficient decrease', ACM Transactions on Mathematical Software 20(3), 286-307.

J. J. Moré and G. Toraldo (1991), 'On the solution of large quadratic programming problems with bound constraints', SIAM Journal on Optimization 1(1), 93-113.

J. J. Moré and S. J. Wright (1993), Optimization Software Guide, number 14 in 'Frontiers in Applied Mathematics', SIAM, Philadelphia, USA.

W. Murray and F. J. Prieto (1995), 'A sequential quadratic programming algorithm using an incomplete solution of the subproblem', SIAM Journal on Optimization 5(3), 590640 .

W. Murray and M. H. Wright (1992), Project Lagrangian methods based on the trajectories of penalty and barrier functions, Numerical Analysis Manuscript 92-01, AT\&T Bell Laboratories.

B. A. Murtagh and M. A. Saunders (1982), 'A projected Lagrangian algorithm and its implementation for sparse non-linear constraints', Mathematical Programming Studies 16, 84-117.

K. G. Murty and S. N. Kabadi (1987), 'Some NP-complete problems in quadratic and nonlinear programming', Mathematical Programming 39(2), 117-129. 
S. G. Nash (1984), 'Newton-type minimization via the Lanczos method', SIAM Journal on Numerical Analysis.

S. G. Nash (2000a), 'A multigrid approach to discretized optimization problems', Optimization Methods and Software 14, 99-116.

S. G. Nash (2000b), 'A survey of truncated-newton methods', Journal of Computational and Applied Mathematics 124, 45-59.

S. G. Nash and J. Nocedal (1991), 'A numerical study of the limited memory BFGS method and the truncated-Newton method for large-scale optimization', SIAM Journal on Optimization 1(3), 358-372.

S. G. Nash and A. Sofer (1990), 'Assessing a search direction within a truncated-Newton method', Operations Research Letters 9(4), 219-221.

Y. Nesterov and A. Nemirovskii (1994), Interior-Point Polynomial Algorithms in Convex Programming, SIAM, Philadelphia, USA.

J. Nocedal (1980), 'Updating quasi-Newton matrices with limited storage', Mathematics of Computation 35, 773-782.

J. Nocedal (1992), 'Theory of algorithms for unconstrained optimization', Acta Numerica 1, 199-242.

J. Nocedal (1997), Large scale unconstrained optimization, in Duff and Watson (1997), pp. 311-338.

J. Nocedal and S. J. Wright (1999), Large sparse numerical optimization, Series in Operations Research, Springer Verlag, Heidelberg, Berlin, New York.

E. O. Omojokun (1989), Trust region algorithms for optimization with nonlinear equality and inequality constraints, PhD thesis, University of Colorado, Boulder, Colorado, USA.

C. C. Paige and M. A. Saunders (1982), 'LSQR: an algorithm for sparse linear equations and sparse least squares', Transactions of the ACM on Mathematical Software 8, 4371.

E. Polak and G. Ribiere (1969), 'Note sur la convergence de méthodes de directions conjuguées', Revue francaise d'informatique et de recherche opérationelle 16-R1, 3543.

B. T. Polyak (1969), 'The conjugate gradient method in extremal problems', U.S.S.R. Computational Mathematics and Mathematical Physics 9, 94-112.

M. J. D. Powell (1977), 'Restart procedures for the conjugate gradient method', Mathematical Programming 12(2), 241-254. 
M. J. D. Powell (1998), 'Direct search algorithms for optimization calculations', Acta Numerica 7, 287-336.

C. J. Price and Ph. L. Toint (2004), Exploiting problem structure in pattern-search methods for unconstrained optimization, Technical Report November, Department of Mathematics and Statistics, University of Canterbury, Christchurch, New Zealand.

R. Pytlak (1998), 'An efficient algorithm for large-scale nonlinear programming problems with simple bounds on the variables', SIAM Journal on Optimization 8(2), 532-560.

A. U. Raghunathan and L. T. Biegler (2003), Interior point methods for Mathematical Programs with Complementarity Constraints (MPCCs), Technical Report, Department of Chemical Engineering, Carnegie Mellon University, Pittsburgh, PA, USA.

F. Rendl and H. Wolkowicz (1997), 'A semidefinite framework for trust region subproblems with applications to large scale minimization', Mathematical Programming 77(2), 273-299.

J. Renegar (2001), A mathematical view of interior-point methods in convex optimization, MPS/SIAM series on Optimization, Society for Industrial and Applied Mathematics, Philadelphia, PA, USA.

S. M. Robinson (1974), 'Perturbed Kuhn-Tucker points and rates of convergence for a class of nonlinear programming algorithms', Mathematical Programming 7(1), 1-16.

M. A. Saunders and J. A. Tomlin (1996), Solving regularized linear programs using barrier methods and KKT systems, Technical Report SOL 96-4, Department of EESOR, Stanford University, Stanford, CA, USA.

H. Scheel and S. Scholtes (2000), 'Mathematical programs with complementarity constraints: Stationarity, optimality, and sensitivity', Mathematics of Operations Research 25, 1-22.

T. Schlick (1993), 'Modified Cholesky factorizations for sparse preconditioners', SIAM Journal on Scientific Computing 14(2), 424-445.

J. A. Scott, Y. Hu and N. I. M. Gould (2004), An evaluation of sparse direct symmetric solvers: an introduction and preliminary findings, Numerical Analysis Group Internal Report 2004-1, Rutherford Appleton Laboratory, Chilton, Oxfordshire, England.

S. Smith and L. Lasdon (1992), 'Solving large sparse nonlinear programs using GRG', ORSA Journal on Computing 4, 1-15.

D. C. Sorensen (1997), 'Minimization of a large-scale quadratic function subject to a spherical constraint', SIAM Journal on Optimization 7(1), 141-161. 
E. Spedicato, ed. (1994), Algorithms for Continuous Optimization: The State of the Art, number 434 in 'NATO ASI Series C: Mathematical and Physical Sciences', Kluwer Academic Publishers, Dordrecht, The Netherlands.

T. Steihaug (1983), 'The conjugate gradient method and trust regions in large scale optimization', SIAM Journal on Numerical Analysis 20(3), 626-637.

A. Tits, A. Wächter, S. Bakhtiari, T. J. Urban and C. T. Lawrence (2003), 'A primaldual interior-point method for nonlinear programming with strong global and local convergence properties', SIAM Journal on Optimization 14(1), 173-199.

M. J. Todd (2001), ‘Semidefinite optimization', Acta Numerica 10, 515-560.

Ph. L. Toint (1981), Towards an efficient sparsity exploiting Newton method for minimization, in Sparse Matrices and Their Uses (I. S. Duff, ed.), Academic Press, London, England, pp. 57-88.

Ph. L. Toint (1987), 'On large scale nonlinear least squares calculations', SIAM Journal on Scientific and Statistical Computing 8(3), 416-435.

Ph. L. Toint (1988), 'Global convergence of a class of trust region methods for nonconvex minimization in Hilbert space', IMA Journal of Numerical Analysis 8, 231-252.

Ph. L. Toint (1996), 'An assessment of non-monotone linesearch techniques for unconstrained optimization', SIAM Journal on Scientific and Statistical Computing 17(3), 725-739.

Ph. L. Toint (1997), 'A non-monotone trust-region algorithm for nonlinear optimization subject to convex constraints', Mathematical Programming 77(1), 69-94.

M. Ulbrich (2004a), 'A multidimensional filter trust-region method for mixed complementarity problems', Talk at ICCOPT 1, Troy, USA.

M. Ulbrich, S. Ulbrich and L. N. Vicente (2004), 'A globally convergence primal-dual interior-point filter method for nonlinear programming', Mathematical Programming, Series B 100(2), 379-410.

S. Ulbrich (2004b), 'On the superlinear local convergence of a filter-SQP method', Mathematical Programming, Series B 100(1), 217-245.

R. J. Vanderbei (1995), 'Symmetric quasi-definite matrices', SIAM Journal on Optimization 5, 100-113.

R. J. Vanderbei (1999), 'LOQO: An interior point code for quadratic programming', Optimization Methods and Software 12, 451-484.

R. J. Vanderbei and D. F. Shanno (1999), 'An interior point algorithm for nonconvex nonlinear programming', Computational Optimization and Applications 13, 231-252. 
S. A. Vavasis (1990), 'Quadratic programming is is NP', Information Processing Letters 36(2), $73-77$.

S. A. Vavasis (1991), Convex quadratic programming, in Nonlinear Optimization: Complexity Issues, Oxford University Press, Oxford, England, pp. 36-75.

A. Wächter (2002), An Interior Point Algorithm for Large-Scale Nonlinear Optimization with Applications in Process Engineering, PhD thesis, Department of Chemical Engineering, Carnegie-Mellon University, Pittsburgh, Pennsylvania, USA.

A. Wächter and L. T. Biegler (2000), 'Failure of global convergence for a class of interior point methods for nonlinear programming', Mathematical Programming 88(3), 565574 .

A. Wächter and L. T. Biegler (2003a), Line search filter methods for nonlinear programming: Local convergence, Technical Report RC23033(W0312-090), T. J. Watson Research Center, Yorktown Heights, NY, USA.

A. Wächter and L. T. Biegler (2003b), Line search filter methods for nonlinear programming: Motivation and global convergence, Technical Report RC23036(W0304-181), T. J. Watson Research Center, Yorktown Heights, NY, USA.

A. Wächter and L. T. Biegler (2004), On the Implementation of an Interior-Point Filter Line-Search Algorithm for Large-Scale Nonlinear Programming, Research Report RC 23149, IBM T. J. Watson Research Center, Yorktown Heights, NY, USA.

M. H. Wright (1992), 'Interior methods for constrained optimization', Acta Numerica 1, 341-407.

S. J. Wright (1997), Primal-Dual Interior-Point Methods, SIAM, Philadelphia, USA.

H. Yamashita, H. Yabe and T. Tanabe (2004), 'A globally and superlinearly convergent primal-dual interior point trust region method for large scale constrained optimization', Mathematical Programming. Online First DOI 10.1007/s10107-004-0508-9.

Y. Ye (1997), Interior Point Algorithms, Theory and Analysis, Wiley-Interscience Series in Discrete Mathematics and Optimization, John Wiley \& Sons, New-York, NY, USA.

E. A. Yildirim and S. J. Wright (2002), 'Warm-start strategies in interior-point methods for linear programming', SIAM Journal on Optimization 12(3), 782-810.

Y. Yuan, ed. (1998), Advances in Nonlinear Programming, Kluwer Academic Publishers, Dordrecht, The Netherlands.

Y. Yuan (2000), 'On the truncated conjugate-gradient method', Mathematical Programming, Series A 87(3), 561-573. 
Y. Zhang (1994), 'On the convergence of a class of infeasible interior-point methods for the horizontal linear complementarity problem', SIAM Journal on Optimization 4(1), 208-227.

C. Zhu, R. H. Byrd, P. Lu and J. Nocedal (1997), 'Algorithm 778. L-BFGS-B: Fortran subroutines for large-scale bound constrained optimization', ACM Transactions on Mathematical Software 23(4), 550-560. 